\title{
ON THE TU-ZENG PERMUTATION TRINOMIAL OF TYPE
}

\author{
$(1 / 4,3 / 4)$
}

\author{
XIANG-DONG HOU
}

\begin{abstract}
Let $q$ be a power of 2. Recently, Tu and Zeng considered trinomials of the form $f(X)=X+a X^{(1 / 4) q^{2}(q-1)}+b X^{(3 / 4) q^{2}(q-1)}$, where $a, b \in \mathbb{F}_{q^{2}}^{*}$. They proved that $f$ is a permutation polynomial of $\mathbb{F}_{q^{2}}$ if $b=a^{2-q}$ and $X^{3}+X+a^{-1-q}$ has no root in $\mathbb{F}_{q}$. In this paper, we show that the above sufficient condition is also necessary.
\end{abstract}

\section{INTRODUCTION}

Let $\mathbb{F}_{q}$ denote the finite field with $q$ elements. A polynomial $f \in \mathbb{F}_{q}[X]$ is called a permutation polynomial $(\mathrm{PP})$ of $\mathbb{F}_{q}$ if it induces a permutation of $\mathbb{F}_{q}$. Consider a particular type of polynomials $f \in \mathbb{F}_{q}[X]$, e.g., binomials, trinomials, etc., to determine the PPs of the given type is to find criteria (in terms of the coefficients of $f$ ) for the equation $f(x)=y$ to have at least (or at most) one solution $x \in \mathbb{F}_{q}$ for each $y \in \mathbb{F}_{q}$. Such questions are usually difficult. Much of the research in this direction have been focusing on trinomials with Niho exponents. These are polynomials of the form

$$
f_{\left(r, s_{1}, s_{2},\right), a, b}(X)=X^{r}\left(1+a X^{s_{1}(q-1)}+b X^{s_{2}(q-1)}\right) \in \mathbb{F}_{q^{2}}[X],
$$

where $r, s_{1}, s_{2}$ are positive integers and $a, b \in \mathbb{F}_{q^{2}}$, and the objective is to determine the conditions on $a$ and $b$ that are necessary and sufficient for $f_{\left(r, s_{1}, s_{2}\right), a, b}$ to be a $\mathrm{PP}$ of $\mathbb{F}_{q^{2}}$. The integers $s_{1}$ and $s_{2}$ can be treated as elements of $\mathbb{Z} /(q+1) \mathbb{Z}$; for example, for even $q,\left(s_{1}, s_{2}\right)=(1 / 4,3 / 4)$ is meaningful. The question has been solved for the following cases of parameters $\left(r, s_{1}, s_{2}\right)$ :

- $\left(r, s_{1}, s_{2}\right)=(1,1,2)[2]$.

- $\left(r, s_{1}, s_{2}\right)=(1,-1 / 2,1 / 2), \operatorname{char} \mathbb{F}_{q}=28$.

- $\left(r, s_{1}, s_{2}\right)=(1,-1,2)$, char $\mathbb{F}_{q}=2,3$ [1, 3, 5, 9].

The purpose of the present paper is to add the case $\left(r, s_{1}, s_{2}\right)=(1,1 / 4,3 / 4)$, char $\mathbb{F}_{q}=2$, to the above short list. Let $q$ be even. In a recent paper [8], Tu and Zeng proved that $f_{(1,1 / 4,3 / 4), a, b}(X)=X\left(1+a X^{(q-1) / 4}+b X^{3(q-1) / 4}\right)\left(a, b \in \mathbb{F}_{q^{2}}^{*}\right)$ is a $\mathrm{PP}$ of $\mathbb{F}_{q^{2}}$ if $b=a^{2-q}$ and $X^{3}+X+a^{-1-q}$ has no root in $\mathbb{F}_{q}$. Note that $f_{(1,1 / 4,3 / 4), a, b}\left(X^{4}\right)=X^{4}\left(1+a X^{q-1}+b X^{3(q-1)}\right)=f_{(4,1,3), a, b}(X)$, and by a suitable substitution $X \mapsto u X, u \in \mathbb{F}_{q^{2}}^{*}$, we may assume that $a \in \mathbb{F}_{q}^{*}$. Therefore, the result of Tu and Zeng can be stated as follows: Let $f(X)=X^{4}\left(1+a X^{q-1}+b X^{3(q-1)}\right)$, where $a \in \mathbb{F}_{q}^{*}$ and $b \in \mathbb{F}_{q^{2}}^{*}$. Then $f$ is a PP of $\mathbb{F}_{q^{2}}$ if $a=b$ and $X^{3}+X+a^{-1}$ has no root in $\mathbb{F}_{q}$. We show that the sufficient condition here is also necessary:

2010 Mathematics Subject Classification. 11T06, 11T55, $14 \mathrm{H} 05$.

Key words and phrases. composition, finite field, permutation polynomial, rational function, resultant. 
Theorem 1.1. Let $q$ be even and $f(X)=X^{4}\left(1+a X^{q-1}+b X^{3(q-1)}\right)$, where $a \in \mathbb{F}_{q}^{*}$ and $b \in \mathbb{F}_{q^{2}}^{*}$. Then $f$ is a PP of $\mathbb{F}_{q^{2}}$ if and only if $a=b$ and $X^{3}+X+a^{-1}$ has no root in $\mathbb{F}_{q}$.

The proof of Theorem 1.1 follows a strategy similar to that of [3. By a wellknown folklore, we see that $f$ is a $\mathrm{PP}$ of $\mathbb{F}_{q^{2}}$ if and only if, essentially, a certain quartic equation in $X$ with coefficients in $\mathbb{F}_{q}(Y)$ has a unique solution $X=x \in \mathbb{F}_{q}$ for every $Y=y \in \mathbb{F}_{q}$. This fact, combined with a theorem by Leonard and Williams on the factorization of quartic polynomials over $\mathbb{F}_{q}$ and a theorem by Hou and Iezzi on composition of rational functions over $\mathbb{F}_{q}$, implies a compositional factorization of a certain rational function in $\mathbb{F}_{q}(Y)$. Comparison of the coefficients in the factorization gives several polynomial equations involving $a$ and $b$. A careful analysis of these equations, mostly done through computerized symbolic computations, concludes that the solutions $(a, b)$ of these equations, filtered by some additional requirements, are precisely those described in Theorem 1.1 .

Theorem 1.1 is proved in three steps, in the remaining three sections, respectively, each with a specific goal. In Section 2, we show that if $f$ is a PP of $\mathbb{F}_{q^{2}}$, then a certain rational function in $X$ and $Y$ has a unique zero $(x, y) \in \mathbb{F}_{q}^{2}$ for every $y \in \mathbb{F}_{q}$. In Section 3, we prove that if $f$ is a $\mathrm{PP}$ of $\mathbb{F}_{q^{2}}$, then $b \in \mathbb{F}_{q}^{*}$. This is the most difficult part of the proof. In Section 4, under the assumption that $b \in \mathbb{F}_{q}^{*}$, we conclude that $f$ is a $\mathrm{PP}$ of $\mathbb{F}_{q^{2}}$ if and only if $a=b$ and $X^{3}+X+a^{-1}$ has no root in $\mathbb{F}_{q}$.

The following two results will be used in the proof of Theorem 1.1

Theorem 1.2 (Leonard and Williams [6], Williams [11]). Let $q$ be even and let and $\alpha_{0}, \alpha_{1}, \alpha_{2} \in \mathbb{F}_{q}$ be such that $\alpha_{0} \alpha_{1} \neq 0$.

(i) $X^{4}+\alpha_{2} X^{2}+\alpha_{1} X+\alpha_{0}$ has a unique root in $\mathbb{F}_{q}$ if and only if $X^{3}+\alpha_{2} X+\alpha_{1}$ is irreducible over $\mathbb{F}_{q}$.

(ii) $X^{3}+\alpha_{2} X+\alpha_{1}$ is irreducible over $\mathbb{F}_{q}$ if and only if $\operatorname{Tr}_{q / 2}\left(1+\alpha_{2}^{3} / \alpha_{1}^{2}\right)=0$ and $X^{6}+\alpha_{1} X^{3}+\alpha_{2}^{3}$ has no root in $\mathbb{F}_{q^{2}}$.

Theorem 1.3 (Hou and Iezzi [4). Let $F(X), G(X) \in \mathbb{F}_{q}(X) \backslash \mathbb{F}_{q}$ be such that $\operatorname{deg} F=d$ and $\operatorname{deg} G=\delta$. If there is a constant $0<\epsilon \leq 1$ such that

$$
\left|\left\{(x, y) \in \mathbb{F}_{q} \times \mathbb{F}_{q}: F(x)=G(y)\right\}\right| \geq q\left(\left\lfloor\frac{\delta}{2}\right\rfloor+\epsilon\right),
$$

and $q \geq(d+\delta)^{4} / \epsilon^{2}$, then $F(X)=G(H(X))$ for some $H \in \mathbb{F}_{q}(X)$.

In our notation, the resultant of two polynomials $P_{1}(X)$ and $P_{2}(X)$ is denoted by $\operatorname{Res}\left(P_{1}, P_{2} ; X\right)$. If $P \in \mathbb{F}_{q}[X]$ and $u \in \mathbb{F}_{q}$ is an unknown element, $P(u)$ is frequently treated as a polynomial in $u$ rather than an element of $\mathbb{F}_{q}$. Therefore the meaning of $\operatorname{Res}\left(P_{1}(u), P_{2}(u) ; u\right)$ is $\left.\operatorname{Res}\left(P_{1}(X), P_{2}(X) ; X\right)\right|_{X=u}$.

\section{INITIAL APPROACH}

From now on, $q$ is even and

$$
f(X)=X^{4}\left(1+a X^{q-1}+b X^{3(q-1)}\right),
$$

where $a \in \mathbb{F}_{q}^{*}$ and $b \in \mathbb{F}_{q^{2}}^{*}$. We assume that $q \geq 2^{13}$. (For $q \leq 2^{12}$, Theorem 1.1 can be verified with a computer.) It is well known [7, 10, 12] that $f$ is a $\mathrm{PP}$ of $\mathbb{F}_{q^{2}}^{*}$ if 
and only if $f_{1}(X):=X^{4}\left(1+a X+b X^{3}\right)^{q-1}$ permutes $\mu_{q+1}:=\left\{x \in \mathbb{F}_{q^{2}}: x^{q+1}=1\right\}$. For $x \in \mu_{q+1}$ with $1+a x+b x^{3} \neq 0$, we have $f_{1}(x)=g(x)$, where

$$
g(X)=\frac{X\left(b^{q}+a X^{2}+X^{3}\right)}{1+a X+b X^{3}} .
$$

Therefore, $f$ is a PP of $\mathbb{F}_{q^{2}}$ if and only if $1+a X+b X^{3}$ has no root in $\mu_{q+1}$ and $g(X)$ permutes $\mu_{q+1}$.

Assume that $1+a X+b X^{3}$ has no root in $\mu_{q+1}$, in particular, $1+a+b \neq 0$. Choose $z \in \mathbb{F}_{q^{2}}$ such that $\operatorname{Tr}_{q^{2} / q}(z)=1$ and let $k=\mathrm{N}_{q^{2} / q}(z)$. Then $z^{2}+z+k=0$ and $\operatorname{Tr}_{q / 2}(k)=1$. Let $\phi(X)=\left(X+z^{q}\right) /(X+z)$, which maps $\mathbb{F}_{q} \cup\{\infty\}$ to $\mu_{q+1}$ bijectively, and let $\psi(X)=(1+a+b)^{q-1} \phi(X)$. We have the following diagram:

$$
\begin{array}{ccccccc}
\mathbb{F}_{q} \cup\{\infty\} & \stackrel{\phi}{\longrightarrow} & \mu_{q+1} & \stackrel{g}{\longrightarrow} & \mu_{q+1} & \stackrel{\psi^{-1}}{\longrightarrow} & \mathbb{F}_{q} \cup\{\infty\} \\
\infty & \longmapsto & 1 & \longmapsto & (1+a+b)^{q-1} & \longmapsto & \infty
\end{array}
$$

where $\psi^{-1}$ denotes the compositional inverse of $\psi$. Therefore, $g(X)$ permutes $\mu_{q+1}$ if and only if $\psi^{-1} \circ g \circ \phi$ permutes $\mathbb{F}_{q}$, that is, if and only if for each $y \in \mathbb{F}_{q}$, there is a unique $x \in \mathbb{F}_{q}$ such that $(g \circ \phi)(x)=\psi(y)$, i.e.,

$$
g\left(\frac{x+z+1}{x+z}\right)=(1+a+b)^{q-1} \frac{y+z+1}{y+z} .
$$

\section{Necessity that $b \in \mathbb{F}_{q}^{*}$}

In this section we prove the following claim:

Claim 3.1. If $f$ is a $P P$ of $\mathbb{F}_{q^{2}}$, then $b \in \mathbb{F}_{q}^{*}$.

The proof of this moderate claim turns out to be quite involved. For the rest of this section, assume that $f$ is a $\mathrm{PP}$ of $\mathbb{F}_{q^{2}}$ and assume to the contrary that $b \notin \mathbb{F}_{q}$.

3.1. From a quartic equation to a system of equations. Let $b_{1}=b+b^{q}$ and $z=b / b_{1}$. Then $b=b_{1} z, \operatorname{Tr}_{q^{2} / q}(z)=1$, and $k:=\mathrm{N}_{q^{2} / q}(z)=b^{1+q} / b_{1}^{2}$. In (2.3), write

$$
g\left(\frac{X+z+1}{X+z}\right)=\frac{A(X)}{B(X)},
$$

where

$$
\begin{aligned}
A(X)= & 1+a z+a z^{2}+a z^{3}+b_{1} z^{3}+z^{4}+a z^{4}+b_{1} z^{5}+\left(a+a z^{2}+b_{1} z^{2}+b_{1} z^{3}\right) X \\
& +\left(a+a z+b_{1} z+b_{1} z^{2}\right) X^{2}+\left(a+b_{1}+b_{1} z\right)+X^{3}+\left(1+a+b_{1}+b_{1} z\right) X^{4}, \\
B(X)= & b_{1} z^{2}+a z^{3}+b_{1} z^{3}+z^{4}+a z^{4}+b_{1} z^{4}+b_{1} z^{5}+\left(b_{1} z+a z^{2}+b_{1} z^{3}\right) X \\
& +\left(a z+b_{1} z+b_{1} z^{2}\right) X^{2}+\left(a+b_{1} z\right) X^{3}+\left(1+a+b_{1} z\right) X^{4} .
\end{aligned}
$$

Since $1+a X+b X^{3}$ has no root in $\mu_{q+1}, B(X)$ has no root in $\mathbb{F}_{q}$. Now (2.3) becomes

$$
\frac{A(x)}{B(x)}=\frac{1+a+b_{1}(z+1)}{1+a+b_{1} z} \cdot \frac{y+z+1}{y+z} .
$$

Using the relation $z^{2}+z+k=0$, we write (3.2) as

$$
C_{4} x^{4}+C_{3}(y) x^{3}+C_{2}(y) x^{2}+C_{1}(y) x+C_{0}(y)=0,
$$

where

$$
C_{4}=a^{2}+a b_{1}+b_{1}^{2} k+b_{1}+1(\neq 0),
$$




$$
\begin{aligned}
C_{3}(Y)= & a^{2}+a b_{1}+a+b_{1}^{2} k+b_{1} Y, \\
C_{2}(Y)= & b_{1}^{2} k+b_{1} k+\left(a^{2}+a+b_{1}^{2} k\right) Y, \\
C_{1}(Y)= & a^{2} k+a b_{1} k+a k+b_{1}^{2} k^{2}+b_{1}^{2} k+b_{1} k+\left(a^{2}+a+b_{1}^{2} k+b_{1} k\right) Y, \\
C_{0}(Y)= & a^{2} k^{2}+a b_{1} k^{2}+a k+b_{1}^{2} k^{3}+b_{1}^{2} k^{2}+k^{2}+k \\
& +\left(a^{2} k+a k+a+b_{1}^{2} k^{2}+b_{1} k+1\right) Y .
\end{aligned}
$$

Recall from Section 2 that for each $y \in \mathbb{F}_{q}$, there is a unique $x \in \mathbb{F}_{q}$ satisfying (3.3). In the above $C_{4} \neq 0$ since otherwise, $\operatorname{Tr}_{q / 2}(k)=\operatorname{Tr}_{q / 2}\left(b_{1}^{-1}+b_{1}^{-2}+a / b_{1}+\left(a / b_{1}\right)^{2}\right)=$ 0 , which is a contradiction. Write $C_{i}=C_{i}(Y)$ and $c_{i}=C_{i}(y), 0 \leq i \leq 4$. Squaring (3.3) and replacing $x$ with $x^{1 / 2}$ give

$$
c_{4}^{2} x^{4}+c_{3}^{2} x^{3}+c_{2}^{2} x^{2}+c_{1}^{2} x+c_{0}^{2}=0 .
$$

Clearly, $C_{3} \neq 0$. We also have $C_{1}^{2} C_{4}+C_{1} C_{2} C_{3}+C_{0} C_{3}^{2} \neq 0$; this is equivalent to saying that $E_{0}, \ldots, E_{3}$, given in (3.8) - (3.11), are not all 0 . In fact, at the end of this subsection, we show that $E_{2}$ and $E_{3}$ are not both 0 . Let $y \in \mathbb{F}_{q}$ be such that $c_{3}\left(c_{1}^{2} c_{4}+c_{1} c_{2} c_{3}+c_{0} c_{3}^{2}\right) \neq 0$. Replacing $x$ be $x+c_{1} / c_{3}$ in (3.4) gives

$$
c_{3}^{4} c_{4}^{2} x^{4}+c_{3}^{6} x^{3}+c_{3}^{4}\left(c_{1} c_{3}+c_{2}^{2}\right) x^{2}+\left(c_{1}^{2} c_{4}+c_{1} c_{2} c_{3}+c_{0} c_{3}^{2}\right)^{2}=0 .
$$

Further replacing $x$ by $1 / x$ in the above gives

$$
x^{4}+\alpha_{2} x^{2}+\alpha_{1} x+\alpha_{0}=0,
$$

where

$$
\begin{aligned}
\alpha_{0} & =\frac{c_{3}^{4} c_{4}^{2}}{\left(c_{1}^{2} c_{4}+c_{1} c_{2} c_{3}+c_{0} c_{3}^{2}\right)^{2}}, \\
\alpha_{1} & =\frac{c_{3}^{6}}{\left(c_{1}^{2} c_{4}+c_{1} c_{2} c_{3}+c_{0} c_{3}^{2}\right)^{2}}, \\
\alpha_{2} & =\frac{c_{3}^{4}\left(c_{1} c_{3}+c_{2}^{2}\right)}{\left(c_{1}^{2} c_{4}+c_{1} c_{2} c_{3}+c_{0} c_{3}^{2}\right)^{2}} .
\end{aligned}
$$

Therefore (3.5) has a unique solution $x$ in $\mathbb{F}_{q}$. By Theorem 1.2, we have

$$
\operatorname{Tr}_{q / 2}\left(1+\frac{\alpha_{2}^{3}}{\alpha_{1}^{2}}\right)=0
$$

i.e.,

$$
\operatorname{Tr}_{q / 2}\left(1+\frac{\left(c_{1} c_{3}+c_{2}^{2}\right)^{3}}{\left(c_{1}^{2} c_{4}+c_{1} c_{2} c_{3}+c_{0} c_{3}^{2}\right)^{2}}\right)=0 .
$$

To recap, for each $y \in \mathbb{F}_{q}$ such that $c_{3}\left(c_{1}^{2} c_{4}+c_{1} c_{2} c_{3}+c_{0} c_{3}^{2}\right) \neq 0$, there are two $x \in \mathbb{F}_{q}$ such that

$$
1+\frac{\left(c_{1} c_{3}+c_{2}^{2}\right)^{3}}{\left(c_{1}^{2} c_{4}+c_{1} c_{2} c_{3}+c_{0} c_{3}^{2}\right)^{2}}=x+x^{2} .
$$

Let $F(Y)=1+\left(C_{1} C_{3}+C_{2}^{2}\right)^{3} /\left(C_{1}^{2} C_{4}+C_{1} C_{2} C_{3}+C_{0} C_{3}^{2}\right)^{2}$ and $G(Y)=Y+Y^{2}$, where $\operatorname{deg} F=d \leq 6$ and $\operatorname{deg} G=\delta=2$. Choose $\epsilon=2^{-1 / 2}$. Then we have

$$
\left|\left\{(y, x): \in \mathbb{F}_{q} \times \mathbb{F}_{q}: F(y)=G(x)\right\}\right| \geq(q-4) \cdot 2=2 q-8>q(1+\epsilon)
$$

and

$$
q \geq 2^{13}=(6+2)^{4} / \epsilon^{2} .
$$


By Theorem 1.3. there exists $H \in \mathbb{F}_{q}(Y)$ such that $F(Y)=G(H(Y))$, i.e.,

$$
1+\frac{\left(C_{1} C_{3}+C_{2}^{2}\right)^{3}}{\left(C_{1}^{2} C_{4}+C_{1} C_{2} C_{3}+C_{0} C_{3}^{2}\right)^{2}}=H(Y)+H(Y)^{2} .
$$

It follows that

$$
H(Y)=\frac{D(Y)}{C_{1}^{2} C_{4}+C_{1} C_{2} C_{3}+C_{0} C_{3}^{2}}
$$

for some $D(Y) \in \mathbb{F}_{q}[Y]$ and (3.6) becomes

$$
\left(C_{1}^{2} C_{4}+C_{1} C_{2} C_{3}+C_{0} C_{3}^{2}\right)^{2}+\left(C_{1} C_{3}+C_{2}^{2}\right)^{3}=D\left(D+C_{1}^{2} C_{4}+C_{1} C_{2} C_{3}+C_{0} C_{3}^{2}\right) .
$$

Since $\operatorname{deg} C_{i} \leq 1$, we have $\operatorname{deg} D \leq 3$. Write

$$
\begin{gathered}
D=\sum_{i=0}^{3} D_{i} Y^{i} \\
C_{1}^{2} C_{4}+C_{1} C_{2} C_{3}+C_{0} C_{3}^{2}=\sum_{i=0}^{3} E_{i} Y^{i}, \\
\left(C_{1}^{2} C_{4}+C_{1} C_{2} C_{3}+C_{0} C_{3}^{2}\right)^{2}+\left(C_{1} C_{3}+C_{2}^{2}\right)^{3}=\sum_{i=0}^{6} F_{i} Y^{i},
\end{gathered}
$$

where

$$
\begin{aligned}
E_{0}= & k\left(a^{5}+a^{4}+a^{3} b_{1}^{2}+a^{3}+a^{2} b_{1}^{2}+a^{2}\right)+k^{3}\left(a b_{1}^{4}+b_{1}^{4}\right) \\
& +k^{2}\left(a b_{1}^{4}+a b_{1}^{2}+b_{1}^{5}+b_{1}^{4}+b_{1}^{3}+b_{1}^{2}\right), \\
E_{1}= & a^{5}+a^{4}+a^{3} b_{1}^{2}+a^{3}+a^{2} b_{1}^{2}+a^{2}+k\left(a^{4} b_{1}+a^{2} b_{1}^{3}+a^{2} b_{1}\right) \\
& +k^{2}\left(a b_{1}^{4}+b_{1}^{5}+b_{1}^{4}+b_{1}^{3}\right)+k^{3} b_{1}^{5},
\end{aligned}
$$

$$
\begin{aligned}
F_{0}= & k^{2}\left(a^{10}+a^{8}+a^{6} b_{1}^{4}+a^{6}+a^{4} b_{1}^{4}+a^{4}\right)+k^{3}\left(a^{12}+a^{10} b_{1}+a^{10}+a^{9} b_{1}^{3}+a^{9} b_{1}\right. \\
& +a^{8} b_{1}^{2}+a^{8}+a^{6} b_{1}^{6}+a^{6} b_{1}^{3}+a^{6} b_{1}+a^{6}+a^{5} b_{1}^{5}+a^{5} b_{1}+a^{4} b_{1}^{7}+a^{4} b_{1}^{6}+a^{4} b_{1}^{3} \\
& \left.+a^{4} b_{1}^{2}+a^{3} b_{1}^{9}+a^{3} b_{1}^{7}+a^{3} b_{1}^{5}+a^{3} b_{1}^{3}\right)+k^{4}\left(a^{8} b_{1}^{3}+a^{8} b_{1}^{2}+a^{4} b_{1}^{5}+a^{4} b_{1}^{4}+a^{4} b_{1}^{3}\right. \\
& \left.+a^{4} b_{1}^{2}+a^{2} b_{1}^{9}+a^{2} b_{1}^{5}+b_{1}^{10}+b_{1}^{8}+b_{1}^{6}+b_{1}^{4}\right)+k^{5}\left(a^{8} b_{1}^{4}+a^{2} b_{1}^{10}+a^{2} b_{1}^{7}+a^{2} b_{1}^{5}\right. \\
& \left.+a^{2} b_{1}^{4}+a b_{1}^{9}+a b_{1}^{5}\right)+k^{6}\left(a^{2} b_{1}^{8}+b_{1}^{9}+b_{1}^{7}+b_{1}^{6}\right)+k^{7}\left(a^{4} b_{1}^{8}+a^{2} b_{1}^{9}+a^{2} b_{1}^{8}+a b_{1}^{11}\right. \\
& \left.+a b_{1}^{9}+b_{1}^{10}+b_{1}^{8}\right)+k^{8}\left(b_{1}^{11}+b_{1}^{10}\right)+k^{9} b_{1}^{12},
\end{aligned}
$$

$$
\begin{aligned}
F_{1}= & k^{2}\left(a^{12}+a^{11} b_{1}+a^{10} b_{1}+a^{10}+a^{8} b_{1}^{2}+a^{8}+a^{7} b_{1}^{3}+a^{7} b_{1}+a^{6} b_{1}^{6}+a^{6} b_{1}^{3}+a^{6} b_{1}\right. \\
& \left.+a^{6}+a^{5} b_{1}^{7}+a^{5} b_{1}^{3}+a^{4} b_{1}^{7}+a^{4} b_{1}^{6}+a^{4} b_{1}^{3}+a^{4} b_{1}^{2}\right)+k^{3}\left(a^{9} b_{1}^{3}+a^{8} b_{1}^{3}+a^{8} b_{1}^{2}\right. \\
& +a^{5} b_{1}^{5}+a^{5} b_{1}^{3}+a^{4} b_{1}^{5}+a^{4} b_{1}^{4}+a^{4} b_{1}^{3}+a^{4} b_{1}^{2}+a^{3} b_{1}^{9}+a^{3} b_{1}^{5}+a^{2} b_{1}^{9}+a^{2} b_{1}^{8}+a^{2} b_{1}^{5} \\
& \left.+a^{2} b_{1}^{4}\right)+k^{4}\left(a^{8} b_{1}^{4}+a^{3} b_{1}^{7}+a^{3} b_{1}^{5}+a^{2} b_{1}^{10}+a^{2} b_{1}^{7}+a^{2} b_{1}^{5}+a^{2} b_{1}^{4}\right)+k^{5}\left(a b_{1}^{9}+a b_{1}^{7}\right. \\
& \left.+b_{1}^{9}+b_{1}^{8}+b_{1}^{7}+b_{1}^{6}\right)+k^{6}\left(a^{4} b_{1}^{8}+a^{3} b_{1}^{9}+a^{2} b_{1}^{9}+a^{2} b_{1}^{8}+b_{1}^{10}+b_{1}^{8}\right) \\
& +k^{7}\left(a b_{1}^{11}+b_{1}^{11}+b_{1}^{10}\right)+k^{8} b_{1}^{12},
\end{aligned}
$$




$$
\begin{aligned}
F_{2}= & a^{10}+a^{8}+a^{6} b_{1}^{4}+a^{6}+a^{4} b_{1}^{4}+a^{4}+k\left(a^{12}+a^{10} b_{1}^{2}+a^{10} b_{1}+a^{10}+a^{9} b_{1}^{3}+a^{9} b_{1}\right. \\
& \left.+a^{8} b_{1}^{3}+a^{8}+a^{7} b_{1}^{5}+a^{7} b_{1}^{3}+a^{6} b_{1}^{3}+a^{6} b_{1}^{2}+a^{6} b_{1}+a^{6}+a^{5} b_{1}^{5}+a^{5} b_{1}\right)+k^{2}\left(a^{12}\right. \\
& +a^{10} b_{1}+a^{10}+a^{9} b_{1}+a^{8} b_{1}^{3}+a^{8} b_{1}^{2}+a^{8}+a^{6} b_{1}^{6}+a^{6} b_{1}^{5}+a^{6} b_{1}^{4}+a^{6} b_{1}^{3}+a^{6} b_{1} \\
& \left.+a^{6}+a^{5} b_{1}^{3}+a^{5} b_{1}+a^{4} b_{1}^{7}+a^{4} b_{1}^{5}+a^{4} b_{1}^{4}+a^{4} b_{1}^{2}+a^{3} b_{1}^{7}+a^{3} b_{1}^{3}\right)+k^{3}\left(a^{8} b_{1}^{4}\right. \\
& +a^{8} b_{1}^{3}+a^{8} b_{1}^{2}+a^{4} b_{1}^{7}+a^{4} b_{1}^{6}+a^{4} b_{1}^{5}+a^{4} b_{1}^{4}+a^{4} b_{1}^{3}+a^{4} b_{1}^{2}+a^{3} b_{1}^{9}+a^{3} b_{1}^{7}+a^{2} b_{1}^{9} \\
& \left.+a^{2} b_{1}^{8}+a^{2} b_{1}^{7}+a^{2} b_{1}^{6}+a b_{1}^{9}+a b_{1}^{5}\right)+k^{4}\left(a^{8} b_{1}^{4}+a^{2} b_{1}^{10}+a^{2} b_{1}^{9}+a^{2} b_{1}^{7}+a^{2} b_{1}^{5}\right. \\
& \left.+a^{2} b_{1}^{4}+a b_{1}^{7}+a b_{1}^{5}+b_{1}^{10}+b_{1}^{9}+b_{1}^{7}\right)+k^{5}\left(a^{4} b_{1}^{8}+a^{2} b_{1}^{10}+a^{2} b_{1}^{9}+a^{2} b_{1}^{8}+a b_{1}^{11}\right. \\
& \left.+a b_{1}^{9}+b_{1}^{10}+b_{1}^{9}+b_{1}^{7}+b_{1}^{6}\right)+k^{6}\left(a^{4} b_{1}^{8}+a^{2} b_{1}^{9}+a^{2} b_{1}^{8}+a b_{1}^{9}+b_{1}^{11}+b_{1}^{10}+b_{1}^{8}\right) \\
& +k^{7}\left(b_{1}^{12}+b_{1}^{11}+b_{1}^{10}\right)+k^{8} b_{1}^{12}
\end{aligned}
$$

$$
\begin{aligned}
F_{3}= & a^{12}+a^{11} b_{1}+a^{10} b_{1}^{2}+a^{10} b_{1}+a^{10}+a^{9} b_{1}^{3}+a^{8} b_{1}^{3}+a^{8}+a^{7} b_{1}^{3}+a^{7} b_{1}+a^{6} b_{1}^{3} \\
& +a^{6} b_{1}^{2}+a^{6} b_{1}+a^{6}+k\left(a^{9} b_{1}^{3}+a^{8} b_{1}^{3}+a^{8} b_{1}^{2}+a^{7} b_{1}^{5}+a^{6} b_{1}^{5}+a^{6} b_{1}^{4}+a^{5} b_{1}^{5}+a^{5} b_{1}^{3}\right. \\
& \left.+a^{4} b_{1}^{5}+a^{4} b_{1}^{4}+a^{4} b_{1}^{3}+a^{4} b_{1}^{2}\right)+k^{2}\left(a^{8} b_{1}^{4}+a^{5} b_{1}^{7}+a^{4} b_{1}^{7}+a^{4} b_{1}^{6}+a^{3} b_{1}^{7}+a^{3} b_{1}^{5}\right. \\
& \left.+a^{2} b_{1}^{7}+a^{2} b_{1}^{6}+a^{2} b_{1}^{5}+a^{2} b_{1}^{4}\right)+k^{3}\left(a^{3} b_{1}^{9}+a^{2} b_{1}^{9}+a^{2} b_{1}^{8}+a b_{1}^{9}+a b_{1}^{7}+b_{1}^{9}+b_{1}^{8}\right. \\
& \left.+b_{1}^{7}+b_{1}^{6}\right)+k^{4}\left(a^{4} b_{1}^{8}+a^{3} b_{1}^{9}+a^{2} b_{1}^{10}+a^{2} b_{1}^{9}+a^{2} b_{1}^{8}+b_{1}^{10}+b_{1}^{8}\right) \\
& +k^{5}\left(a b_{1}^{11}+b_{1}^{11}+b_{1}^{10}\right)+k^{6} b_{1}^{12},
\end{aligned}
$$

$F_{4}=a^{12}+a^{10} b_{1}^{2}+a^{10} b_{1}+a^{9} b_{1}+a^{8} b_{1}^{3}+a^{8} b_{1}^{2}+a^{7} b_{1}^{3}+a^{6} b_{1}^{3}+a^{6} b_{1}^{2}+a^{6} b_{1}+a^{5} b_{1}^{3}$

$$
\begin{aligned}
& +a^{5} b_{1}+a^{4} b_{1}^{2}+a^{4}+k\left(a^{12}+a^{10} b_{1}+a^{10}+a^{9} b_{1}^{3}+a^{9} b_{1}+a^{8} b_{1}^{3}+a^{8}+a^{6} b_{1}^{5}\right. \\
& \left.+a^{6} b_{1}^{4}+a^{6} b_{1}^{3}+a^{6} b_{1}+a^{6}+a^{5} b_{1}^{5}+a^{5} b_{1}+a^{4} b_{1}^{5}+a^{4} b_{1}^{4}+a^{3} b_{1}^{5}+a^{3} b_{1}^{3}\right) \\
& +k^{2}\left(a^{8} b_{1}^{4}+a^{8} b_{1}^{3}+a^{8} b_{1}^{2}+a^{4} b_{1}^{7}+a^{4} b_{1}^{6}+a^{4} b_{1}^{5}+a^{4} b_{1}^{4}+a^{4} b_{1}^{3}+a^{4} b_{1}^{2}+a^{3} b_{1}^{7}\right. \\
& \left.+a^{2} b_{1}^{7}+a^{2} b_{1}^{6}+a^{2} b_{1}^{4}+a b_{1}^{7}+a b_{1}^{5}+b_{1}^{4}\right)+k^{3}\left(a^{8} b_{1}^{4}+a^{2} b_{1}^{9}+a^{2} b_{1}^{8}+a^{2} b_{1}^{7}+a^{2} b_{1}^{5}\right. \\
& \left.+a^{2} b_{1}^{4}+a b_{1}^{9}+a b_{1}^{5}+b_{1}^{9}+b_{1}^{8}+b_{1}^{7}+b_{1}^{6}\right)+k^{4}\left(a^{4} b_{1}^{8}+a^{2} b_{1}^{10}+a^{2} b_{1}^{9}+a b_{1}^{9}+b_{1}^{9}\right. \\
& \left.+b_{1}^{8}+b_{1}^{7}+b_{1}^{6}\right)+k^{5}\left(a^{4} b_{1}^{8}+a^{2} b_{1}^{9}+a^{2} b_{1}^{8}+a b_{1}^{11}+a b_{1}^{9}+b_{1}^{11}+b_{1}^{8}\right) \\
& +k^{6}\left(b_{1}^{12}+b_{1}^{11}+b_{1}^{10}\right)+k^{7} b_{1}^{12}
\end{aligned}
$$

$$
\begin{aligned}
F_{5}= & a^{12}+a^{11} b_{1}+a^{10} b_{1}+a^{10}+a^{8} b_{1}^{2}+a^{8}+a^{7} b_{1}^{3}+a^{7} b_{1}+a^{6} b_{1}^{3}+a^{6} b_{1}+a^{6}+a^{5} b_{1}^{3} \\
& +a^{4} b_{1}^{3}+a^{4} b_{1}^{2}+k\left(a^{9} b_{1}^{3}+a^{8} b_{1}^{3}+a^{8} b_{1}^{2}+a^{5} b_{1}^{5}+a^{5} b_{1}^{3}+a^{4} b_{1}^{5}+a^{4} b_{1}^{4}+a^{4} b_{1}^{3}\right. \\
& \left.+a^{4} b_{1}^{2}+a^{3} b_{1}^{5}+a^{2} b_{1}^{5}+a^{2} b_{1}^{4}\right)+k^{2}\left(a^{8} b_{1}^{4}+a^{3} b_{1}^{7}+a^{3} b_{1}^{5}+a^{2} b_{1}^{7}+a^{2} b_{1}^{5}+a^{2} b_{1}^{4}\right) \\
& +k^{3}\left(a b_{1}^{9}+a b_{1}^{7}+b_{1}^{9}+b_{1}^{8}+b_{1}^{7}+b_{1}^{6}\right)+k^{4}\left(a^{4} b_{1}^{8}+a^{3} b_{1}^{9}+a^{2} b_{1}^{9}+a^{2} b_{1}^{8}+b_{1}^{10}+b_{1}^{8}\right) \\
& +k^{5}\left(a b_{1}^{11}+b_{1}^{11}+b_{1}^{10}\right)+k^{6} b_{1}^{12},
\end{aligned}
$$

$$
\begin{aligned}
F_{6}= & a^{12}+a^{10} b_{1}+a^{10}+a^{9} b_{1}+a^{8}+a^{6} b_{1}^{3}+a^{6} b_{1}+a^{6}+a^{5} b_{1}^{3}+a^{5} b_{1}+a^{4} b_{1}^{3}+a^{3} b_{1}^{3} \\
& +b_{1}^{4}+a^{2} b_{1}^{4}+k\left(a^{8} b_{1}^{3}+a^{8} b_{1}^{2}+a^{4} b_{1}^{5}+a^{4} b_{1}^{4}+a^{4} b_{1}^{3}+a^{4} b_{1}^{2}+a^{2} b_{1}^{5}+a^{2} b_{1}^{4}\right) \\
& +k^{2}\left(a^{8} b_{1}^{4}+a^{2} b_{1}^{7}+a^{2} b_{1}^{5}+a^{2} b_{1}^{4}+a b_{1}^{7}+a b_{1}^{5}+b_{1}^{6}\right)+k^{3}\left(b_{1}^{9}+b_{1}^{8}+b_{1}^{7}+b_{1}^{6}\right)
\end{aligned}
$$




$$
+k^{4}\left(a^{4} b_{1}^{8}+a^{2} b_{1}^{9}+a^{2} b_{1}^{8}+a b_{1}^{9}+b_{1}^{8}\right)+k^{5}\left(b_{1}^{11}+b_{1}^{10}\right)+k^{6} b_{1}^{12} .
$$

Then (3.7) becomes

$$
\left(\sum_{i=0}^{3} D_{i} Y^{i}\right)\left(\sum_{j=0}^{3}\left(D_{j}+E_{j}\right) Y^{j}\right)=\sum_{i=0}^{6} F_{i} Y^{i} .
$$

Comparing the coefficients in the above gives

$$
\begin{aligned}
& D_{3}^{2}+D_{3} E_{3}=F_{6}, \\
& D_{3} E_{2}+D_{2} E_{3}=F_{5}, \\
& D_{2}^{2}+D_{3} E_{1}+D_{2} E_{2}+D_{1} E_{3}=F_{4}, \\
& D_{3} E_{0}+D_{2} E_{1}+D_{1} E_{2}+D_{0} E_{3}=F_{3}, \\
& D_{1}^{2}+D_{2} E_{0}+D_{1} E_{1}+D_{0} E_{2}=F_{2}, \\
& D_{1} E_{0}+D_{0} E_{1}=F_{1}, \\
& D_{0}^{2}+D_{0} E_{0}=F_{0} .
\end{aligned}
$$

The above is a system of seven polynomial equations in seven unknowns $D_{0}, D_{1}, D_{2}$, $D_{3}, a, b_{1}, k$ in $\mathbb{F}_{q}$. In what follows, we show that the above system does not allow a solution with $a, b_{1}, k \in \mathbb{F}_{q}^{*}$ such that $\operatorname{Tr}_{q / 2}(k)=1$ and $1+a X+b X^{3}$ has no root in $\mu_{q+1}$. This proves Claim 3.1 by contradiction.

Before proceeding, we mention that $E_{2}$ and $E_{3}$ are not both 0 , a claim that was used earlier in this subsection to transform (3.4) to (3.5). We have $\operatorname{Res}\left(E_{2}, E_{3} ; a\right)=$ $b_{1}^{17} k^{2} \neq 0$, hence the claim.

3.2. Nonsolvability of the system. $1^{\circ}$ We temporarily assume that $E_{3} \neq 0$. By (3.20) - (3.22),

$$
\begin{aligned}
& D_{2}=\left(D_{3} E_{2}+F_{5}\right) / E_{3}, \\
& D_{1}=\left(D_{2}^{2}+D_{3} E_{1}+D_{2} E_{2}+F_{4}\right) / E_{3}, \\
& D_{0}=\left(D_{3} E_{0}+D_{2} E_{1}+D_{1} E_{2}+F_{3}\right) / E_{3} .
\end{aligned}
$$

By these substitutions, the remaining equations in (3.19) - (3.25) become

$D_{3}^{2}+D_{3} E_{3}+F_{6}=0$

$D_{3}^{4} E_{2}^{4}+D_{3}^{2} E_{1}^{2} E_{3}^{4}+D_{3}^{2} E_{1} E_{2}^{2} E_{3}^{3}+D_{3} E_{1}^{2} E_{3}^{5}+D_{3} E_{1} E_{2}^{2} E_{3}^{4}+D_{3} E_{2}^{4} E_{3}^{3}+E_{0} E_{3}^{5} F_{5}$

$+E_{1} E_{3}^{5} F_{4}+E_{1} E_{3}^{3} F_{5}^{2}+E_{2}^{3} E_{3}^{3} F_{5}+E_{2}^{2} E_{3}^{4} F_{4}+E_{2} E_{3}^{5} F_{3}+E_{3}^{6} F_{2}+E_{3}^{4} F_{4}^{2}+F_{5}^{4}=0$,

$D_{3}^{2} E_{0} E_{2}^{2} E_{3}+D_{3}^{2} E_{1} E_{2}^{3}+D_{3} E_{0} E_{2}^{2} E_{3}^{2}+D_{3} E_{1} E_{2}^{3} E_{3}+E_{0} E_{2} E_{3}^{2} F_{5}+E_{0} E_{3}^{3} F_{4}$

$+E_{0} E_{3} F_{5}^{2}+E_{1}^{2} E_{3}^{2} F_{5}+E_{1} E_{2}^{2} E_{3} F_{5}+E_{1} E_{2} E_{3}^{2} F_{4}+E_{1} E_{2} F_{5}^{2}+E_{1} E_{3}^{3} F_{3}+E_{3}^{4} F_{1}=0$,

$D_{3}^{4} E_{2}^{6}+D_{3}^{2} E_{0}^{2} E_{3}^{6}+D_{3}^{2} E_{0} E_{2}^{3} E_{3}^{4}+D_{3}^{2} E_{2}^{6} E_{3}^{2}+D_{3} E_{0}^{2} E_{3}^{7}+D_{3} E_{0} E_{2}^{3} E_{3}^{5}+E_{0} E_{1} E_{3}^{6} F_{5}$

$+E_{0} E_{2}^{2} E_{3}^{5} F_{5}+E_{0} E_{2} E_{3}^{6} F_{4}+E_{0} E_{2} E_{3}^{4} F_{5}^{2}+E_{0} E_{3}^{7} F_{3}+E_{1}^{2} E_{3}^{4} F_{5}^{2}+E_{2}^{4} E_{3}^{2} F_{5}^{2}$

$+E_{2}^{2} E_{3}^{4} F_{4}^{2}+E_{2}^{2} F_{5}^{4}+E_{3}^{8} F_{0}+E_{3}^{6} F_{3}^{2}=0$. 
In fact, (3.26) - (3.29) are linear combinations of (3.19) - (3.25) with coefficients in $\mathbb{F}_{2}\left[D_{0}, \ldots, D_{3}, E_{0}, \ldots, E_{3}, F_{0}, \ldots, F_{6}\right]$. Hence they hold even if $E_{3}=0$. Thus we remove the assumption that $E_{3} \neq 0$. Subtracting (3.27) - (3.29) by suitable multiples of (3.26) allows us to eliminate $D_{3}$, resulting in three equations in $E_{i}$ 's and $F_{j}$ 's:

$$
\begin{aligned}
H_{1}:= & E_{0} E_{3}^{5} F_{5}+E_{1}^{2} E_{3}^{4} F_{6}+E_{1} E_{2}^{2} E_{3}^{3} F_{6}+E_{1} E_{3}^{5} F_{4}+E_{1} E_{3}^{3} F_{5}^{2}+E_{2}^{4} E_{3}^{2} F_{6}+E_{2}^{4} F_{6}^{2} \\
& +E_{2}^{3} E_{3}^{3} F_{5}+E_{2}^{2} E_{3}^{4} F_{4}+E_{2} E_{3}^{5} F_{3}+E_{3}^{6} F_{2}+E_{3}^{4} F_{4}^{2}+F_{5}^{4}=0,
\end{aligned}
$$

$$
\begin{aligned}
H_{2}:= & E_{0} E_{2}^{2} E_{3} F_{6}+E_{0} E_{2} E_{3}^{2} F_{5}+E_{0} E_{3}^{3} F_{4}+E_{0} E_{3} F_{5}^{2}+E_{1}^{2} E_{3}^{2} F_{5}+E_{1} E_{2}^{3} F_{6} \\
& +E_{1} E_{2}^{2} E_{3} F_{5}+E_{1} E_{2} E_{3}^{2} F_{4}+E_{1} E_{2} F_{5}^{2}+E_{1} E_{3}^{3} F_{3}+E_{3}^{4} F_{1}=0,
\end{aligned}
$$

$$
\begin{aligned}
H_{3}:= & E_{0}^{2} E_{3}^{6} F_{6}+E_{0} E_{1} E_{3}^{6} F_{5}+E_{0} E_{2}^{3} E_{3}^{4} F_{6}+E_{0} E_{2}^{2} E_{3}^{5} F_{5}+E_{0} E_{2} E_{3}^{6} F_{4} \\
& +E_{0} E_{2} E_{3}^{4} F_{5}^{2}+E_{0} E_{3}^{7} F_{3}+E_{1}^{2} E_{3}^{4} F_{5}^{2}+E_{2}^{6} F_{6}^{2}+E_{2}^{4} E_{3}^{2} F_{5}^{2}+E_{2}^{2} E_{3}^{4} F_{4}^{2} \\
& +E_{2}^{2} F_{5}^{4}+E_{3}^{8} F_{0}+E_{3}^{6} F_{3}^{2}=0 .
\end{aligned}
$$

$H_{1}, H_{2}, H_{3}$ are polynomials in $a, b_{1}, k$ :

$$
\begin{aligned}
& H_{1}=C_{4}^{4} h_{1}, \\
& H_{2}=C_{4}^{5}\left(a^{2}+b_{1}^{2} k\right)^{2} h_{2}, \\
& H_{3}=C_{4}^{4} h_{3},
\end{aligned}
$$

where $h_{1}, h_{2}, h_{3}$ are given in Appendix (A1) - A3). We note that $\operatorname{deg}_{k} h_{1}=20$, $\operatorname{deg}_{k} h_{2}=10$, and $\operatorname{deg}_{k} h_{3}=24$.

We claim that $a^{2}+b_{1}^{2} k \neq 0$. Assume to the contrary that $k=a^{2} / b_{1}^{2}$. Then

$$
\begin{aligned}
& h_{1}=a^{7} b_{1}^{10}\left(a^{3}+b_{1}+a^{2} b_{1}\right), \\
& h_{3}=a^{9} b_{1}^{12}\left(a^{5}+b_{1}+a^{4} b_{1}+b_{1}^{3}+a^{4} b_{1}^{3}\right),
\end{aligned}
$$

and

$$
\operatorname{Res}\left(a^{3}+b_{1}+a^{2} b_{1}, a^{5}+b_{1}+a^{4} b_{1}+b_{1}^{3}+a^{4} b_{1}^{3} ; b_{1}\right)=a^{3}(1+a)^{4}\left(1+a+a^{3}\right)^{2} .
$$

Thus $(1+a)\left(1+a+a^{3}\right)=0$. If $1+a=0$, then $a^{3}+b_{1}+a^{2} b_{1}=1 \neq 0$, which is a contradiction. If $1+a+a^{3}=0$, then from $a^{3}+b_{1}+a^{2} b_{1}=0$ we have $b_{1}=a^{3} /(1+a)^{2}=a^{-3}$, hence $k=a^{2} / b_{1}^{2}=a^{8}$. Note that $a \in \mathbb{F}_{2^{3}} \subset \mathbb{F}_{q}$ and $\operatorname{Tr}_{2^{3} / 2}(a)=0$. Thus $\operatorname{Tr}_{q / 2}(k)=\operatorname{Tr}_{q / 2}(a)=0$, which is a contradiction. Thus the claim is proved.

Now by (3.33) - (3.35), we have $h_{1}=h_{2}=h_{3}=0$. Using $h_{2}$ to reduce the degree of $k$ in $h_{1}$ and $h_{3}$, we find that $h_{1} \equiv b_{1}^{8} h_{1}^{\prime}\left(\bmod h_{2}\right)$ and $h_{3} \equiv b_{1}^{10} h_{3}^{\prime}\left(\bmod h_{2}\right)$, where $h_{1}^{\prime}$ and $h_{3}^{\prime}$, given in (A4) and (A5), are of degree 9 in $k$. To recap, we now have three polynomial equations in $a, b_{1}, k$ :

$$
h_{1}^{\prime}=0, \quad h_{2}=0, \quad h_{3}^{\prime}=0 .
$$

$2^{\circ}$ We have

$$
\begin{aligned}
\operatorname{Res}\left(h_{1}^{\prime}, h_{2} ; a\right) & =b_{1}^{208} k^{37}\left(1+b_{1}+b_{1}^{2} k\right)^{2}\left(1+b_{1}^{2} k\right)^{2} S_{1}^{6} S_{2}, \\
\operatorname{Res}\left(h_{2}, h_{3}^{\prime} ; a\right) & =b_{1}^{272} k^{69}\left(1+b_{1}+b_{1}^{2} k\right)^{2} S_{1}^{8} S_{3}, \\
\operatorname{Res}\left(h_{1}^{\prime}, h_{2} ; b_{1}\right) & =a^{108}(1+a)^{108} k^{131} T_{1}^{6} T_{2},
\end{aligned}
$$




$$
\operatorname{Res}\left(h_{2}, h_{3}^{\prime} ; b_{1}\right)=a^{126}(1+a)^{152} k^{158} T_{1}^{8} T_{3},
$$

where $S_{1}, S_{2}, S_{3}, T_{1}, T_{2}, T_{3}$ are given in (A6) - (A11). In (3.37) and (3.38), $1+b_{1}+$ $b_{1}^{2} k \neq 0$ since $\operatorname{Tr}_{q / 2}(k)=1$.

Case 1. Assume $a=1$. We have $\left.h_{1}^{\prime}\right|_{a=1}=b_{1}^{9} k^{3} h_{1}^{\prime \prime}$ and $\left.h_{2}\right|_{a=1}=b_{1}^{9} k^{3} h_{3}^{\prime \prime}$, where

$$
\begin{aligned}
h_{1}^{\prime \prime}= & b_{1}^{10} k^{6}+b_{1}^{11} k^{5}+b_{1}^{9} k^{5}+b_{1}^{8} k^{5}+b_{1}^{10} k^{4}+b_{1}^{9} k^{4}+b_{1}^{9} k^{3}+b_{1}^{7} k^{2} \\
& +b_{1}^{6} k^{2}+b_{1}^{2} k^{2}+b_{1} k+k+b_{1}^{4}+b_{1}^{3}+b_{1}^{2}, \\
h_{2}^{\prime \prime}= & b_{1}^{11} k^{7}+b_{1}^{9} k^{6}+b_{1}^{8} k^{5}+b_{1}^{8} k^{4}+b_{1}^{7} k^{3}+b_{1}^{6} k^{3}+b_{1}^{3} k^{3}+b_{1}^{5} k^{2} \\
& +b_{1}^{4} k^{2}+b_{1}^{2} k+b_{1} k^{2}+k+b_{1}^{2} .
\end{aligned}
$$

It turns out that $\operatorname{Res}\left(h_{1}^{\prime \prime}, h_{2}^{\prime \prime} ; b_{1}\right)=k^{66} \neq 0$, which is a contradiction.

Case 2. Assume that $a \neq 1$ but $T_{1}=0$, i.e., $k=a^{-2}+a^{-1}+1+a^{3}+a^{4}+a^{6}=$ $(1+a)^{5}\left(1+a^{2}+a^{3}\right) / a^{2}$. We have

$$
\begin{aligned}
& \left.h_{1}^{\prime}\right|_{T_{1}=0}=a^{-16}(1+a)^{4}\left(a+b_{1}+a b_{1}+a^{2} b_{1}+a^{3} b_{1}\right) h_{1}^{*}, \\
& \left.h_{2}\right|_{T_{1}=0}=a^{-20}(1+a)^{4}\left(a+b_{1}+a b_{1}+a^{2} b_{1}+a^{3} b_{1}\right) h_{2}^{*}, \\
& \left.h_{3}^{\prime}\right|_{T_{1}=0}=a^{-17}(1+a)^{4}\left(a+b_{1}+a b_{1}+a^{2} b_{1}+a^{3} b_{1}\right) h_{3}^{*} .
\end{aligned}
$$

where $h_{1}^{*}, h_{2}^{*}, h_{3}^{*}$ are given in (A12) - A14).

First assume that $a+b_{1}+a b_{1}+a^{2} b_{1}+a^{3} b_{1} \neq 0$. Then $h_{1}^{*}=h_{2}^{*}=h_{3}^{*}=0$. However, we find that as a polynomial in $b_{1}$,

$$
\operatorname{gcd}\left(\operatorname{Res}\left(h_{1}^{*}, h_{2}^{*} ; a\right), \operatorname{Res}\left(h_{2}^{*}, h_{3}^{*} ; a\right)\right)=b_{1}^{1720},
$$

which dose not have a nonzero root. This is a contradiction.

Now assume that $a+b_{1}+a b_{1}+a^{2} b_{1}+a^{3} b_{1}=0$, i.e., $b_{1}=a /(1+a)^{3}$. Making substitutions $k=(1+a)^{5}\left(1+a^{2}+a^{3}\right) / a^{2}$ and $b_{1}=a /(1+a)^{3}$ gives $E_{3}=0$.

Case 3. Assume that $(1+a) T_{1} \neq 0$. Then by (3.39) and (3.40), $T_{2}=T_{3}=0$. We find that

$$
\begin{aligned}
& \operatorname{Res}\left(T_{2}, T_{3} ; a\right)=T_{4}^{2} T_{5}^{2} T_{6}^{2} T_{7}^{2}, \\
& \operatorname{Res}\left(T_{2}, T_{3} ; k\right)=(1+a)^{44} T_{8}^{2} T_{9}^{2} T_{10}^{2} T_{11}^{2}
\end{aligned}
$$

where

$$
\begin{aligned}
T_{4}= & k^{7}+k^{6}+k^{5}+k^{3}+k^{2}+k+1, \\
T_{5}= & k^{13}+k^{12}+k^{11}+k^{10}+k^{8}+k^{7}+k^{6}+k^{5}+k^{4}+k^{3}+1, \\
T_{6}= & k^{21}+k^{20}+k^{19}+k^{18}+k^{16}+k^{14}+k^{12}+k^{11}+k^{10}+k^{8}+k^{7}+k^{6}+k^{5} \\
& +k^{4}+k^{3}+k^{2}+1, \\
T_{7}= & k^{49}+k^{46}+k^{44}+k^{40}+k^{39}+k^{38}+k^{37}+k^{36}+k^{35}+k^{30}+k^{24}+k^{22}+k^{15} \\
& +k^{14}+k^{12}+k^{10}+k^{8}+k^{3}+k^{2}+k+1, \\
T_{8}= & a^{7}+a^{3}+1, \\
T_{9}= & a^{13}+a^{11}+a^{10}+a^{5}+a^{3}+a+1, \\
T_{10}= & a^{21}+a^{20}+a^{18}+a^{14}+a^{11}+a^{8}+a^{7}+a^{4}+1, \\
T_{11}= & a^{49}+a^{48}+a^{47}+a^{44}+a^{43}+a^{42}+a^{40}+a^{38}+a^{37}+a^{36}+a^{33}+a^{31}+a^{26}
\end{aligned}
$$




$$
+a^{25}+a^{22}+a^{20}+a^{19}+a^{18}+a^{17}+a^{16}+a^{15}+a^{8}+a^{6}+a^{5}+1 .
$$

In the above $T_{7} \neq 0$ since $\operatorname{Tr}_{q / 2}(k)=1$.

Case 3.1. Assume in (3.37) that $\left(1+b_{1}^{2} k\right) S_{1} \neq 0$. Then by (3.37) and (3.38), $S_{2}=S_{3}=0$. We find that, as a polynomial in $k$,

$$
\operatorname{gcd}\left(\operatorname{Res}\left(S_{2}, S_{3} ; b_{1}\right), \operatorname{Res}\left(T_{2}, T_{3} ; a\right)\right)=1,
$$

which is a contradiction. (Recall that $\operatorname{Res}\left(T_{2}, T_{3} ; a\right)$ is given in (3.45).)

Case 3.2. Assume in (3.37) that $\left(1+b_{1}^{2} k\right) S_{1}=0$.

First assume that $1+b_{1}^{2} k=0$. We have $\left.h_{1}^{\prime}\right|_{k=b_{1}^{-2}}=a h_{1}^{\dagger},\left.h_{2}\right|_{k=b_{1}^{-2}}=a h_{2}^{\dagger}$, $\left.h_{3}^{\prime}\right|_{k=b_{1}^{-2}}=a h_{3}^{\dagger}$, where

$$
\begin{aligned}
h_{1}^{\dagger}= & a^{21}+a^{19} b_{1}^{4}+a^{19} b_{1}^{2}+a^{17}+a^{16} b_{1}^{5}+a^{16} b_{1}^{3}+a^{14} b_{1}^{5}+a^{14} b_{1}^{3}+a^{13} b_{1}^{6}+a^{13} b_{1}^{4} \\
& +a^{13}+a^{12} b_{1}^{5}+a^{11} b_{1}^{6}+a^{11} b_{1}^{2}+a^{10} b_{1}^{5}+a^{9} b_{1}^{6}+a^{9} b_{1}^{4}+a^{9}+a^{8} b_{1}^{7}+a^{8} b_{1}^{3} \\
& +a^{6} b_{1}^{7}+a^{6} b_{1}^{5}+a^{6} b_{1}^{3}+a^{4} b_{1}^{5}+a^{2} b_{1}^{5}+a b_{1}^{4}+b_{1}^{7}, \\
h_{2}^{\dagger}= & a^{19}+a^{17}+a^{16} b_{1}+a^{14} b_{1}^{3}+a^{12} b_{1}^{3}+a^{11} b_{1}^{4}+a^{11}+a^{10} b_{1}^{3}+a^{9} b_{1}^{4}+a^{9}+a^{8} b_{1}^{5} \\
& +a^{8} b_{1}^{3}+a^{8} b_{1}+a^{7} b_{1}^{4}+a^{6} b_{1}^{5}+a^{5} b_{1}^{4}+a^{3} b_{1}^{4}+a b_{1}^{4}+b_{1}^{5}, \\
h_{3}^{\dagger}= & a^{23} b_{1}^{2}+a^{23}+a^{22} b_{1}^{3}+a^{21} b_{1}^{4}+a^{21} b_{1}^{2}+a^{21}+a^{20} b_{1}^{5}+a^{20} b_{1}+a^{19} b_{1}^{4}+a^{19} \\
& +a^{18} b_{1}^{5}+a^{18} b_{1}^{3}+a^{17} b_{1}^{6}+a^{17} b_{1}^{4}+a^{17} b_{1}^{2}+a^{17}+a^{16} b_{1}^{7}+a^{16} b_{1}^{5}+a^{16} b_{1} \\
& +a^{15} b_{1}^{8}+a^{15} b_{1}^{6}+a^{15} b_{1}^{4}+a^{15} b_{1}^{2}+a^{15}+a^{14} b_{1}^{5}+a^{13} b_{1}^{6}+a^{13} b_{1}^{4}+a^{13} b_{1}^{2} \\
& +a^{13}+a^{12} b_{1}^{9}+a^{12} b_{1}^{7}+a^{12} b_{1}^{5}+a^{12} b_{1}^{3}+a^{12} b_{1}+a^{11} b_{1}^{8}+a^{11} b_{1}^{4}+a^{11} \\
& +a^{10} b_{1}^{7}+a^{10} b_{1}^{5}+a^{9} b_{1}^{10}+a^{9} b_{1}^{8}+a^{9} b_{1}^{4}+a^{9} b_{1}^{2}+a^{9}+a^{8} b_{1}^{9}+a^{8} b_{1}^{7}+a^{8} b_{1}^{5} \\
& +a^{8} b_{1}^{3}+a^{8} b_{1}+a^{7} b_{1}^{10}+a^{7} b_{1}^{4}+a^{5} b_{1}^{6}+a^{4} b_{1}^{9}+a^{4} b_{1}^{7}+a^{4} b_{1}^{5}+a^{3} b_{1}^{6}+a^{3} b_{1}^{4} \\
& +a b_{1}^{10}+a b_{1}^{8}+a b_{1}^{6}+a b_{1}^{4}+b_{1}^{9}+b_{1}^{5} .
\end{aligned}
$$

Moreover,

$$
\begin{aligned}
\operatorname{Res}\left(h_{1}^{\dagger}, h_{2}^{\dagger} ; a\right) & =b_{1}^{119}\left(1+b_{1}+b_{1}^{2}\right)^{10}\left(1+b_{1}+b_{1}^{4}\right)^{2}, \\
\operatorname{Res}\left(h_{2}^{\dagger}, h_{3}^{\dagger} ; a\right) & =b_{1}^{135}\left(1+b_{1}+b_{1}^{2}\right)^{8}\left(1+b_{1}+b_{1}^{2}+b_{1}^{4}+b_{1}^{6}\right)^{2}, \\
\operatorname{Res}\left(h_{1}^{\dagger}, h_{2}^{\dagger} ; b_{1}\right) & =a^{65}(1+a)^{54}\left(1+a+a^{2}\right)^{10}\left(1+a+a^{4}\right)^{2}\left(1+a^{3}+a^{4}\right)^{8} .
\end{aligned}
$$

By (3.47) and (3.48), we have $1+b_{1}+b_{1}^{2}=0$. Thus $k=b_{1}^{-2} \in \mathbb{F}_{2^{2}}$. Since $\operatorname{Tr}_{q / 2}(k)=1,\left[\mathbb{F}_{q}: \mathbb{F}_{2^{2}}\right]$ is odd. Then by (3.49), we have $1+a+a^{2}=0$. Therefore, $a=b_{1}$ or $b_{1}^{-1}$. However,

$$
\left.h_{2}^{\dagger}\right|_{a=b_{1}^{-1}}=b_{1}^{-19}\left(1+b_{1}+b_{1}^{3}\right)^{2}\left(1+b_{1}^{2}+b_{1}^{3}\right)^{2}\left(1+b_{1}^{3}+b_{1}^{6}\right)^{2} \neq 0 .
$$

So we must have $a=b_{1}$. Recall that $\operatorname{Tr}_{q^{2} / q}(b)=b_{1}$ and $\mathrm{N}_{q^{2} / q}(b)=b_{1}^{2} k=1$. Hence $b^{2}+b_{1} b+1=0$. Then $b^{-1} \in \mu_{q+1}$ is a root of $1+a X+b X^{3}$, which is a contradiction.

Now assume that $S_{1}=0$. Recall that in (3.45), $T_{7} \neq 0$. Hence $T_{4} T_{5} T_{6}=0$. Then $\left[\mathbb{F}_{2}(k): \mathbb{F}_{2}\right]$ is odd. Since $\operatorname{Tr}_{q / 2}(k)=1,\left[\mathbb{F}_{q}: \mathbb{F}_{2}\right]$ is also odd.

Case 3.2.1. Assume that $T_{4}=0$. We have

$$
\operatorname{Res}\left(S_{1}, T_{4} ; k\right)=T_{12} T_{13} T_{14}
$$


where

$$
\begin{aligned}
T_{12}= & b_{1}^{7}+b_{1}^{6}+b_{1}^{5}+b_{1}^{4}+b_{1}^{3}+b_{1}^{2}+1 \\
T_{13}= & b_{1}^{14}+b_{1}^{13}+b_{1}^{12}+b_{1}^{11}+b_{1}^{10}+b_{1}^{8}+1, \\
T_{14}= & b_{1}^{35}+b_{1}^{33}+b_{1}^{31}+b_{1}^{29}+b_{1}^{28}+b_{1}^{27}+b_{1}^{26}+b_{1}^{25}+b_{1}^{24}+b_{1}^{23}+b_{1}^{21}+b_{1}^{20} \\
& \quad+b_{1}^{17}+b_{1}^{14}+b_{1}^{13}+b_{1}^{12}+b_{1}^{9}+b_{1}^{8}+b_{1}^{7}+b_{1}+1 .
\end{aligned}
$$

Since $\left[\mathbb{F}_{q}: \mathbb{F}_{2}\right]$ is odd, $T_{13} \neq 0$. Thus $T_{12} T_{14}=0$.

First assume that $T_{12}=0$, i.e., $T_{12}$ is the minimal polynomial of $b_{1}$ over $\mathbb{F}_{2}$. Treat $S_{1}$ and $T_{4}$ as polynomials in $k$ over $\mathbb{F}_{2}\left(b_{1}\right)$, where $\operatorname{deg}_{k} S_{1}=3$ and $\operatorname{deg}_{k} T_{4}=7$. Applying the Euclidean algorithm to $S_{1}$ and $T_{4}$ gives some $r(k) \in \mathbb{F}_{2}\left(b_{1}\right)[k]$ such that $r(k) \in \mathbb{F}_{2}\left(b_{1}\right) S_{1}+\mathbb{F}_{2}\left(b_{1}\right) T_{4}$ and $\operatorname{deg} r(k)=1$; solving $r(k)=0$ gives

$$
k=b_{1}+b_{1}^{3}+b_{1}^{4} .
$$

Now $h_{1}^{\prime}, h_{2}, h_{3}^{\prime}$ can be expressed as polynomials in $a$ and $b_{1}$ which are further reduced modulo $T_{12}$. We find that, as a polynomial in $a$,

$$
\operatorname{gcd}\left(\operatorname{Res}\left(h_{1}^{\prime}, h_{2} ; b_{1}\right), \operatorname{Res}\left(T_{2}, T_{3} ; k\right)\right)=1+a .
$$

(Recall that $\operatorname{Res}\left(T_{2}, T_{3} ; k\right)$ is given in (3.46).) Since $a \neq 1$ (by Case 1 ), we have a contradiction.

Next, assume that $T_{14}=0$, i.e., $T_{14}$ is the minimal polynomial of $b_{1}$ over $\mathbb{F}_{2}$. By the same computation, we have

$$
k=b_{1}^{2}+b_{1}^{4}+b_{1}^{6}+b_{1}^{7}+b_{1}^{10}+b_{1}^{11}+b_{1}^{18}+b_{1}^{21}+b_{1}^{22}+b_{1}^{24}+b_{1}^{25}+b_{1}^{26}+b_{1}^{27}+b_{1}^{30} .
$$

and, as a polynomial in $a$,

$$
\operatorname{gcd}\left(\operatorname{Res}\left(h_{1}^{\prime}, h_{2} ; b_{1}\right), \operatorname{Res}\left(T_{2}, T_{3} ; k\right)\right)=(1+a)^{3},
$$

which is a contradiction.

Case 3.2.2. Assume that $T_{5}=0$. We have

$$
\operatorname{Res}\left(S_{1}, T_{5} ; k\right)=T_{15} T_{16} T_{17}
$$

where

$$
\begin{aligned}
T_{15}= & b_{1}^{13}+b_{1}^{12}+b_{1}^{6}+b_{1}^{5}+b_{1}^{4}+b_{1}^{3}+b_{1}^{2}+b_{1}+1 \\
T_{16}= & b_{1}^{13}+b_{1}^{12}+b_{1}^{10}+b_{1}^{9}+b_{1}^{5}+b_{1}^{3}+b_{1}^{2}+b_{1}+1, \\
T_{17}= & b_{1}^{78}+b_{1}^{76}+b_{1}^{75}+b_{1}^{74}+b_{1}^{72}+b_{1}^{69}+b_{1}^{68}+b_{1}^{67}+b_{1}^{66}+b_{1}^{65}+b_{1}^{64}+b_{1}^{63}+b_{1}^{62} \\
& +b_{1}^{61}+b_{1}^{60}+b_{1}^{59}+b_{1}^{58}+b_{1}^{55}+b_{1}^{54}+b_{1}^{53}+b_{1}^{52}+b_{1}^{51}+b_{1}^{50}+b_{1}^{49}+b_{1}^{46} \\
& +b_{1}^{44}+b_{1}^{41}+b_{1}^{39}+b_{1}^{38}+b_{1}^{36}+b_{1}^{35}+b_{1}^{32}+b_{1}^{31}+b_{1}^{30}+b_{1}^{29}+b_{1}^{28}+b_{1}^{26}+b_{1}^{25} \\
& +b_{1}^{22}+b_{1}^{20}+b_{1}^{19}+b_{1}^{18}+b_{1}^{15}+b_{1}^{14}+b_{1}^{13}+b_{1}^{9}+b_{1}^{6}+b_{1}^{5}+b_{1}^{2}+b_{1}+1 .
\end{aligned}
$$

Since $\left[\mathbb{F}_{q}: \mathbb{F}_{2}\right]$ is odd, $T_{17} \neq 0$. Thus $T_{15} T_{16}=0$. We now go through the same computation as in Case 3.2 .1 (with $T_{4}$ replaced by $T_{5}$ ):

When $T_{15}=0$, we have

$$
k=b_{1}^{3}+b_{1}^{6}+b_{1}^{8}+b_{1}^{9}
$$

and, as a polynomial in $a$,

$$
\operatorname{gcd}\left(\operatorname{Res}\left(h_{1}^{\prime}, h_{2} ; b_{1}\right), \operatorname{Res}\left(T_{2}, T_{3} ; k\right)\right)=1,
$$

which is a contradiction. 
When $T_{16}=0$, we have

$$
k=b_{1}^{2}+b_{1}^{3}+b_{1}^{4}+b_{1}^{6}+b_{1}^{11}
$$

and, as a polynomial in $a$,

$$
\operatorname{gcd}\left(\operatorname{Res}\left(h_{1}^{\prime}, h_{2} ; b_{1}\right), \operatorname{Res}\left(T_{2}, T_{3} ; k\right)\right)=1+a,
$$

which is a contradiction.

Case 3.2.3. Assume that $T_{6}=0$. We have

$$
\operatorname{Res}\left(S_{1}, T_{6} ; k\right)=T_{18} T_{19} T_{20},
$$

where

$$
\begin{aligned}
T_{18}= & b_{1}^{21}+b_{1}^{19}+b_{1}^{18}+b_{1}^{17}+b_{1}^{15}+b_{1}^{13}+b_{1}^{12}+b_{1}^{10}+b_{1}^{9}+b_{1}^{8}+b_{1}^{7}+b_{1}^{6}+1, \\
T_{19}= & b_{1}^{21}+b_{1}^{20}+b_{1}^{19}+b_{1}^{18}+b_{1}^{17}+b_{1}^{15}+b_{1}^{14}+b_{1}^{8}+b_{1}^{7}+b_{1}^{6}+b_{1}^{4}+b_{1}^{3}+1, \\
T_{20}= & b_{1}^{126}+b_{1}^{125}+b_{1}^{124}+b_{1}^{122}+b_{1}^{120}+b_{1}^{119}+b_{1}^{117}+b_{1}^{116}+b_{1}^{114}+b_{1}^{112}+b_{1}^{109} \\
& +b_{1}^{105}+b_{1}^{104}+b_{1}^{103}+b_{1}^{100}+b_{1}^{99}+b_{1}^{95}+b_{1}^{93}+b_{1}^{90}+b_{1}^{88}+b_{1}^{87}+b_{1}^{86}+b_{1}^{85} \\
& +b_{1}^{84}+b_{1}^{80}+b_{1}^{79}+b_{1}^{72}+b_{1}^{71}+b_{1}^{70}+b_{1}^{69}+b_{1}^{68}+b_{1}^{67}+b_{1}^{64}+b_{1}^{63}+b_{1}^{60}+b_{1}^{59} \\
& +b_{1}^{56}+b_{1}^{52}+b_{1}^{50}+b_{1}^{47}+b_{1}^{45}+b_{1}^{43}+b_{1}^{39}+b_{1}^{37}+b_{1}^{34}+b_{1}^{33}+b_{1}^{32}+b_{1}^{27}+b_{1}^{26} \\
& +b_{1}^{20}+b_{1}^{19}+b_{1}^{15}+b_{1}^{13}+b_{1}^{12}+b_{1}^{6}+b_{1}^{5}+b_{1}^{4}+b_{1}+1 .
\end{aligned}
$$

Since $\left[\mathbb{F}_{q}: \mathbb{F}_{2}\right]$ is odd, $T_{20} \neq 0$. Thus $T_{18} T_{19}=0$. Again, we go through the same computation as in Case 3.2.1 (with $T_{4}$ replaced by $T_{6}$ ):

When $T_{18}=0$, we have

$$
k=1+b_{1}+b_{1}^{2}+b_{1}^{3}+b_{1}^{5}+b_{1}^{6}+b_{1}^{8}+b_{1}^{11}+b_{1}^{12}+b_{1}^{14}+b_{1}^{15}+b_{1}^{16}+b_{1}^{18},
$$

and, as a polynomial in $a$,

$$
\operatorname{gcd}\left(\operatorname{Res}\left(h_{1}^{\prime}, h_{2} ; b_{1}\right), \operatorname{Res}\left(T_{2}, T_{3} ; k\right)\right)=1+a,
$$

which is a contradiction.

When $T_{19}=0$, we have

$$
k=b_{1}^{8}+b_{1}^{9}+b_{1}^{10}+b_{1}^{12}+b_{1}^{16}+b_{1}^{17}+b_{1}^{18}+b_{1}^{19},
$$

and, as a polynomial in $a$,

$$
\operatorname{gcd}\left(\operatorname{Res}\left(h_{1}^{\prime}, h_{2} ; b_{1}\right), \operatorname{Res}\left(T_{2}, T_{3} ; k\right)\right)=1,
$$

which is a contradiction.

Summary: Only Case 2 is possible, and in that case we have $a \neq 1$ and

$$
k=(1+a)^{5}\left(1+a^{2}+a^{3}\right) / a^{2}, \quad b_{1}=a /(1+a)^{3}, \quad E_{3}=0 .
$$

$3^{\circ}$ Since $E_{3}=0$, (3.19) - (3.25) become

$$
\begin{aligned}
& D_{3}^{2}=F_{6}, \\
& D_{3} E_{2}=F_{5}, \\
& D_{2}^{2}+D_{3} E_{1}+D_{2} E_{2}=F_{4}, \\
& D_{3} E_{0}+D_{2} E_{1}+D_{1} E_{2}=F_{3}, \\
& D_{1}^{2}+D_{2} E_{0}+D_{1} E_{1}+D_{0} E_{2}=F_{2}, \\
& D_{1} E_{0}+D_{0} E_{1}=F_{1},
\end{aligned}
$$




$$
D_{0}^{2}+D_{0} E_{0}=F_{0} .
$$

We assume that $E_{0} \neq 0$ temporarily. By (3.54) - (3.56),

$$
\begin{aligned}
& D_{1}=\left(D_{0} E_{1}+F_{1}\right) / E_{0}, \\
& D_{2}=\left(D_{1}^{2}+D_{1} E_{1}+D_{0} E_{2}+F_{2}\right) / E_{0}, \\
& D_{3}=\left(D_{2} E_{1}+D_{1} E_{2}+F_{3}\right) / E_{0} .
\end{aligned}
$$

By these substitutions, the remaining equations of (3.51) - (3.57) become

$$
\begin{aligned}
& D_{0}^{4} E_{1}^{6}+D_{0}^{2} E_{0}^{2} E_{1}^{6}+E_{0}^{8} F_{6}+E_{0}^{6} F_{3}^{2}+E_{0}^{4} E_{1}^{2} F_{2}^{2} \\
& +E_{0}^{4} E_{2}^{2} F_{1}^{2}+E_{0}^{2} E_{1}^{4} F_{1}^{2}+E_{1}^{2} F_{1}^{4}=0, \\
& D_{0}^{2} E_{1}^{3} E_{2}+D_{0} E_{0} E_{1}^{3} E_{2}+E_{0}^{4} F_{5}+E_{0}^{3} E_{2} F_{3}+E_{0}^{2} E_{1} E_{2} F_{2} \\
& +E_{0}^{2} E_{2}^{2} F_{1}+E_{0} E_{1}^{2} E_{2} F_{1}+E_{1} E_{2} F_{1}^{2}=0, \\
& D_{0}^{4} E_{1}^{4}+D_{0}^{2} E_{0}^{4} E_{2}^{2}+D_{0}^{2} E_{0}^{3} E_{1}^{2} E_{2}+D_{0} E_{0}^{5} E_{2}^{2}+D_{0} E_{0}^{4} E_{1}^{2} E_{2} \\
& +D_{0} E_{0}^{3} E_{1}^{4}+E_{0}^{6} F_{4}+E_{0}^{5} E_{1} F_{3}+E_{0}^{5} E_{2} F_{2}+E_{0}^{4} E_{1}^{2} F_{2} \\
& +E_{0}^{4} F_{2}^{2}+E_{0}^{3} E_{1}^{3} F_{1}+E_{0}^{3} E_{2} F_{1}^{2}+F_{1}^{4}=0, \\
& D_{0}^{2}+D_{0} E_{0}+F_{0}=0 .
\end{aligned}
$$

In fact, (3.58) - (3.61) are linear combinations of (3.51) - (3.57) with coefficients in $\mathbb{F}_{2}\left[D_{0}, \ldots, D_{3}, E_{0}, E_{1}, E_{2}, F_{0}, \ldots, F_{6}\right]$. Hence (3.58) - (3.61) hold even if $E_{0}=0$. Thus we remove the assumption that $E_{0} \neq 0$. Subtracting (3.58) - (3.60) by suitable multiples of (3.61) allows us to eliminate $D_{0}$, resulting in three equations in $E_{i}$ 's and $F_{j}$ 's:

$$
\begin{aligned}
L_{1}:= & E_{0}^{8} F_{6}+E_{0}^{6} F_{3}^{2}+E_{0}^{4} E_{1}^{2} F_{2}^{2}+E_{0}^{4} E_{2}^{2} F_{1}^{2}+E_{0}^{2} E_{1}^{4} F_{1}^{2}+E_{1}^{6} F_{0}^{2}+E_{1}^{2} F_{1}^{4}=0, \\
L_{2}:= & E_{0}^{4} F_{5}+E_{0}^{3} E_{2} F_{3}+E_{0}^{2} E_{1} E_{2} F_{2}+E_{0}^{2} E_{2}^{2} F_{1}+E_{0} E_{1}^{2} E_{2} F_{1}+E_{1}^{3} E_{2} F_{0} \\
& +E_{1} E_{2} F_{1}^{2}=0, \\
L_{3}:= & E_{0}^{6} F_{4}+E_{0}^{5} E_{1} F_{3}+E_{0}^{5} E_{2} F_{2}+E_{0}^{4} E_{1}^{2} F_{2}+E_{0}^{4} E_{2}^{2} F_{0}+E_{0}^{4} F_{2}^{2}+E_{0}^{3} E_{1}^{3} F_{1} \\
& +E_{0}^{3} E_{1}^{2} E_{2} F_{0}+E_{0}^{3} E_{2} F_{1}^{2}+E_{0}^{2} E_{1}^{4} F_{0}+E_{1}^{4} F_{0}^{2}+F_{1}^{4}=0 .
\end{aligned}
$$

$L_{1}, L_{2}, L_{3}$ are polynomials in $a, b_{1}, k$. Using (3.50), we may express them in terms of $a$ :

$$
\begin{aligned}
& L_{1}=\frac{a^{24}\left(1+a^{2}+a^{3}\right)^{6}\left(1+a+a^{9}\right)^{2}}{(1+a)^{40}}, \\
& L_{2}=\frac{a^{15}\left(1+a^{2}+a^{3}\right)^{3}\left(1+a+a^{9}\right)}{(1+a)^{25}}, \\
& L_{3}=\frac{a^{16}\left(1+a^{2}+a^{3}\right)^{5}\left(1+a^{3}+a^{4}\right)\left(1+a^{4}+a^{5}+a^{6}+a^{7}+a^{16}+a^{19}+a^{20}+a^{21}\right)}{(1+a)^{36}} .
\end{aligned}
$$

It follows that $1+a^{2}+a^{3}=0$. But then, by (3.50),

$$
k=\frac{(1+a)^{5}\left(1+a^{2}+a^{3}\right)}{a^{2}}=0,
$$

which is a contradiction.

This completes the proof of Claim 3.1 


\section{Completion of the Proof}

Form now on we assume that $b \in \mathbb{F}_{q}^{*}$.

$1^{\circ}$ We claim that if $f$ is a PP of $\mathbb{F}_{q^{2}}$, then $a=b$. Assume to the contrary that $a \neq b$. Choose $k \in \mathbb{F}_{q}$ such that $\operatorname{Tr}_{q / 2}(k)=1$ and let $z \in \mathbb{F}_{q^{2}}$ be such that $z^{2}+z+k=0$. We go through the computations in Section 3 again. However, since $b \in \mathbb{F}_{q}^{*}$, the computations are simpler. For (3.1) we have

$$
g\left(\frac{X+z+1}{X+z}\right)=\frac{A(X)}{B(X)}
$$

where

$$
\begin{aligned}
A(X)=1 & +a z+a z^{2}+a z^{3}+b z^{3}+z^{4}+a z^{4}+b z^{4}+\left(a+a z^{2}+b z^{2}\right) X \\
& +(a+a z+b z) X^{2}+(a+b) X^{3}+(1+a+b) X^{4}, \\
B(X)= & b z+b z^{2}+a z^{3}+b z^{3}+z^{4}+a z^{4}+b z^{4}+\left(b+a z^{2}+b z^{2}\right) X \\
& +(b+a z+b z) X^{2}+(a+b) X^{3}+(1+a+b) X^{4} .
\end{aligned}
$$

Equation (3.2) becomes

$$
\frac{A(x)}{B(x)}=\frac{y+z+1}{y+z}
$$

which can be written as

$$
C_{4} x^{4}+C_{3} x^{3}+C_{2}(y) x^{2}+C_{1}(y) x+C_{0}(y)=0,
$$

where

$$
\begin{aligned}
C_{4} & =1+a+b(\neq 0), \\
C_{3} & =a+b(\neq 0), \\
C_{2}(Y) & =b+(a+b) Y, \\
C_{1}(Y) & =b+a k+b k+(a+b) Y, \\
C_{0}(Y) & =k+b k+k^{2}+a k^{2}+b k^{2}+(1+a k+b k) Y .
\end{aligned}
$$

We have

$$
C_{1}^{2} C_{4}+C_{1} C_{2} C_{3}+C_{0} C_{3}^{2}=b^{2}+a^{2} k+b^{2} k+(a+b)^{2} Y+(a+b)^{2} Y^{2} \neq 0,
$$

hence $C_{3}\left(C_{1}^{2} C_{4}+C_{1} C_{2} C_{3}+C_{0} C_{3}^{2}\right) \neq 0$. For (3.8) - (3.18), we have

$$
\begin{aligned}
E_{0}= & b^{2}+\left(a^{2}+b^{2}\right) k, \\
E_{1}= & a^{2}+b^{2}, \\
E_{2}= & a^{2}+b^{2}, \\
E_{3}= & 0, \\
F_{0}= & a^{3} b^{3}+b^{4}+\left(a^{4} b^{2}+a^{2} b^{4}\right) k+\left(a^{4}+a^{5} b+b^{4}+a b^{5}\right) k^{2} \\
& +\left(a^{6}+a^{4} b^{2}+a^{2} b^{4}+b^{6}\right) k^{3}, \\
F_{1}= & a^{4} b^{2}+a^{2} b^{4}+\left(a^{6}+a^{4} b^{2}+a^{2} b^{4}+b^{6}\right) k^{2}, \\
F_{2}= & a^{4}+a^{5} b+a^{4} b^{2}+b^{4}+a^{2} b^{4}+a b^{5}+\left(a^{6}+a^{4} b^{2}+a^{2} b^{4}+b^{6}\right) k \\
& +\left(a^{6}+a^{4} b^{2}+a^{2} b^{4}+b^{6}\right) k^{2}, \\
F_{3}= & a^{6}+a^{4} b^{2}+a^{2} b^{4}+b^{6},
\end{aligned}
$$




$$
\begin{aligned}
& F_{4}=a^{4}+a^{6}+a^{5} b+a^{4} b^{2}+b^{4}+a^{2} b^{4}+a b^{5}+b^{6}+\left(a^{6}+a^{4} b^{2}+a^{2} b^{4}+b^{6}\right) k, \\
& F_{5}=a^{6}+a^{4} b^{2}+a^{2} b^{4}+b^{6} \\
& F_{6}=a^{6}+a^{4} b^{2}+a^{2} b^{4}+b^{6} .
\end{aligned}
$$

Since $E_{3}=0$, by (3.51) and (3.52), we have $D_{3}^{2}=F_{6}$ and $D_{3} E_{2}=F_{5}$, which give $D_{3}=(a+b)^{3}$ and $(a+b)^{5}=(a+b)^{6}$. Then $(a+b)(a+b+1)=0$, which is a contradiction. Thus the claim is proved.

$2^{\circ}$ Since $a=b$, we have $C_{4}=1, C_{3}=0, C_{2}=a, C_{1}=a, C_{0}=k+a k+k^{2}+Y$. Now (3.4) becomes

$$
x^{4}+a^{2} x^{2}+a^{2} x+\left(k+a k+k^{2}+y\right)^{2}=0 .
$$

We observe that

(4.2) has a unique solution $x$ in $\mathbb{F}_{q}$

$$
\begin{aligned}
& \left.\Leftrightarrow X^{3}+a^{2} X+a^{2} \text { is irreducible over } \mathbb{F}_{q} \text { (Theorem } 1.2\right) \\
& \Leftrightarrow(X / a)^{3}+X / a+1 / a \text { has no root in } \mathbb{F}_{q} \\
& \Leftrightarrow X^{3}+X+1 / a \text { has no root in } \mathbb{F}_{q} .
\end{aligned}
$$

This completes the proof for both necessity and sufficiency parts of the Theorem 1.1

\section{REFERENCES}

[1] D. Bartoli, On a conjecture about a class of permutation trinomials, Finite Fields Appl. 52 (2018), $30-50$

[2] X. Hou, Determination of a type of permutation trinomials over finite fields, II, Finite Fields Appl. 35 (2015), 16 - 35.

[3] X. Hou, On a class of permutation trinomials in characteristic 2, Cryptography and Communications, published online December 7, 2018.

[4] X. Hou and A. Iezzi, An application of the Hasse-Weil bound to rational functions over finite Fields, preprint.

[5] X. Hou, Z. Tu, X. Zeng, Determination of a class of permutation trinomials in characteristic three, arXiv:1811.11949

[6] P. A. Leonard and K. S. Williams, Quartics over GF $\left(2^{n}\right)$, Proc. Amer. Math. Soc. 36 (1972), $347-350$

[7] Y. H. Park and J. B. Lee, Permutation polynomials and group permutation polynomials, Bull. Austral. Math. Soc. 63 (2001), $67-74$.

[8] Z. Tu and X. Zeng, Two classes of permutation trinomials with Niho exponents, Finite Fields Appl. 53 (2018), 99 - 112.

[9] Z. Tu, X. Zeng, C. Li, T. Helleseth, Permutation polynomials of the form $\left(x^{p^{m}}-x+\delta\right)^{s}+L(x)$ over the finite field $\mathbb{F}_{p^{2 m}}$ of odd characteristic, Finite Fields Appl. 34 (2015), 20 - 35.

[10] Q. Wang, Cyclotomic mapping permutation polynomials over finite fields, in Sequences, Subsequences, and Consequences, S.W. Golomb, G. Gong, T. Helleseth, H.-Y. Song, (Eds.), pp. 119 - 128, Lecture Notes in Comput. Sci., vol. 4893, Springer, Berlin, 2007.

[11] K. S. Williams, Note on cubics over $\mathrm{GF}\left(2^{n}\right)$ and $\mathrm{GF}\left(3^{n}\right)$, J. Number Theory 7 (1975), 361 -365 .

[12] M. E. Zieve, On some permutation polynomials over $\mathbb{F}_{q}$ of the form $x^{r} h\left(x^{(q-1) / d}\right)$, Proc. Amer. Math. Soc. 137 (2009), 2209 - 2216.

\section{APPENDIX}

Gathered here are the lengthy intermediate computational results that were produced and used in the proof of Theorem 1.1. 
In 3.33 - 3.35,

(A1)

$$
\begin{aligned}
& h_{1}=a^{40}+a^{36}+b_{1}^{3} a^{33}+b_{1}^{4} a^{32}+b_{1}^{3} a^{32}+b_{1}^{4} a^{30}+b_{1}^{3} a^{30}+b_{1}^{4} a^{29}+b_{1}^{6} a^{28}+b_{1}^{4} a^{28} \\
& +b_{1}^{3} a^{28}+b_{1}^{6} a^{27}+b_{1}^{5} a^{27}+b_{1}^{5} a^{26}+b_{1}^{3} a^{26}+b_{1}^{7} a^{25}+b_{1}^{5} a^{25}+b_{1}^{4} a^{25}+b_{1}^{8} a^{24}+b_{1}^{7} a^{24} \\
& +b_{1}^{6} a^{24}+b_{1}^{4} a^{24}+b_{1}^{3} a^{24}+a^{24}+b_{1}^{5} a^{23}+b_{1}^{8} a^{22}+b_{1}^{7} a^{22}+b_{1}^{6} a^{22}+b_{1}^{5} a^{22}+b_{1}^{3} a^{22} \\
& +b_{1}^{7} a^{21}+b_{1}^{6} a^{21}+b_{1}^{5} a^{21}+b_{1}^{4} a^{21}+b_{1}^{8} a^{20}+b_{1}^{4} a^{20}+b_{1}^{3} a^{20}+a^{20}+b_{1}^{9} a^{19}+b_{1}^{8} a^{19} \\
& +b_{1}^{6} a^{19}+b_{1}^{5} a^{19}+b_{1}^{9} a^{18}+b_{1}^{5} a^{18}+b_{1}^{3} a^{18}+b_{1}^{9} a^{17}+b_{1}^{7} a^{17}+b_{1}^{5} a^{17}+b_{1}^{4} a^{17}+b_{1}^{3} a^{17} \\
& +b_{1}^{8} a^{16}+b_{1}^{7} a^{16}+b_{1}^{6} a^{16}+b_{1}^{10} a^{15}+b_{1}^{5} a^{15}+b_{1}^{10} a^{14}+b_{1}^{8} a^{14}+b_{1}^{7} a^{14}+b_{1}^{6} a^{14}+b_{1}^{5} a^{14} \\
& +b_{1}^{4} a^{14}+b_{1}^{10} a^{13}+b_{1}^{7} a^{13}+b_{1}^{6} a^{13}+b_{1}^{5} a^{13}+b_{1}^{10} a^{12}+b_{1}^{8} a^{12}+b_{1}^{6} a^{12}+b_{1}^{10} a^{11}+b_{1}^{9} a^{11} \\
& +b_{1}^{8} a^{11}+b_{1}^{10} a^{10}+b_{1}^{9} a^{10}+b_{1}^{10} a^{9}+b_{1}^{9} a^{9}+b_{1}^{10} a^{8}+b_{1}^{40} k^{20}+b_{1}^{36} k^{18}+b_{1}^{36} k^{17}+\left(b_{1}^{36}\right. \\
& \left.+a b_{1}^{35}+b_{1}^{35}+a^{8} b_{1}^{32}+a^{4} b_{1}^{32}\right) k^{16}+\left(b_{1}^{34}+b_{1}^{33}\right) k^{15}+\left(b_{1}^{34}+a^{2} b_{1}^{32}+a b_{1}^{32}+b_{1}^{32}\right. \\
& \left.+a^{2} b_{1}^{31}+b_{1}^{31}\right) k^{14}+\left(a b_{1}^{32}+a b_{1}^{31}+a^{4} b_{1}^{30}+a^{4} b_{1}^{29}+b_{1}^{29}\right) k^{13}+\left(b_{1}^{32}+a b_{1}^{31}+b_{1}^{31}\right. \\
& +a^{4} b_{1}^{30}+a^{3} b_{1}^{30}+a^{3} b_{1}^{29}+a^{2} b_{1}^{29}+a b_{1}^{29}+b_{1}^{29}+a^{6} b_{1}^{28}+a^{5} b_{1}^{28}+a^{4} b_{1}^{28}+a b_{1}^{28}+b_{1}^{28} \\
& \left.+a^{6} b_{1}^{27}+a^{4} b_{1}^{27}+a^{2} b_{1}^{27}+b_{1}^{27}+b_{1}^{24}\right) k^{12}+\left(b_{1}^{29}+a^{2} b_{1}^{28}+a b_{1}^{28}+a^{2} b_{1}^{27}+a b_{1}^{27}+b_{1}^{27}\right. \\
& \left.+a^{8} b_{1}^{26}+a^{8} b_{1}^{25}+b_{1}^{25}\right) k^{11}+\left(a^{2} b_{1}^{28}+b_{1}^{28}+a^{2} b_{1}^{27}+b_{1}^{27}+a^{8} b_{1}^{26}+a^{4} b_{1}^{26}+a^{2} b_{1}^{26}\right. \\
& +a b_{1}^{26}+b_{1}^{26}+a^{4} b_{1}^{25}+a^{3} b_{1}^{25}+a^{2} b_{1}^{25}+a b_{1}^{25}+a^{10} b_{1}^{24}+a^{9} b_{1}^{24}+a^{8} b_{1}^{24}+a b_{1}^{24}+b_{1}^{24} \\
& \left.+a^{10} b_{1}^{23}+a^{8} b_{1}^{23}+a^{2} b_{1}^{23}+b_{1}^{23}+b_{1}^{20}\right) k^{10}+\left(a^{2} b_{1}^{26}+a b_{1}^{26}+b_{1}^{26}+a^{4} b_{1}^{25}+a^{3} b_{1}^{25}\right. \\
& +a b_{1}^{25}+b_{1}^{25}+a^{9} b_{1}^{24}+a^{6} b_{1}^{24}+a^{5} b_{1}^{24}+a^{2} b_{1}^{24}+a b_{1}^{24}+a^{9} b_{1}^{23}+a^{6} b_{1}^{23}+a^{5} b_{1}^{23}+a^{4} b_{1}^{23} \\
& \left.+a b_{1}^{23}+a^{12} b_{1}^{22}+a^{12} b_{1}^{21}+a^{8} b_{1}^{21}+a^{4} b_{1}^{21}+b_{1}^{21}+b_{1}^{20}\right) k^{9}+\left(a^{3} b_{1}^{25}+a^{2} b_{1}^{25}+a b_{1}^{25}\right. \\
& +b_{1}^{25}+a^{8} b_{1}^{24}+a^{6} b_{1}^{24}+a^{4} b_{1}^{24}+a^{3} b_{1}^{24}+a^{2} b_{1}^{24}+a b_{1}^{24}+b_{1}^{24}+a^{9} b_{1}^{23}+a^{8} b_{1}^{23}+a^{6} b_{1}^{23} \\
& +a^{5} b_{1}^{23}+a^{2} b_{1}^{23}+a b_{1}^{23}+a^{12} b_{1}^{22}+a^{11} b_{1}^{22}+a^{8} b_{1}^{22}+a^{6} b_{1}^{22}+a^{5} b_{1}^{22}+a^{3} b_{1}^{22}+a^{11} b_{1}^{21} \\
& +a^{10} b_{1}^{21}+a^{9} b_{1}^{21}+a^{7} b_{1}^{21}+a^{6} b_{1}^{21}+a^{5} b_{1}^{21}+a^{3} b_{1}^{21}+a^{2} b_{1}^{21}+a b_{1}^{21}+b_{1}^{21}+a^{14} b_{1}^{20} \\
& +a^{13} b_{1}^{20}+a^{12} b_{1}^{20}+a^{9} b_{1}^{20}+a^{8} b_{1}^{20}+a^{5} b_{1}^{20}+a^{4} b_{1}^{20}+a b_{1}^{20}+a^{14} b_{1}^{19}+a^{12} b_{1}^{19}+a^{10} b_{1}^{19} \\
& \left.+a^{8} b_{1}^{19}+a^{6} b_{1}^{19}+a^{4} b_{1}^{19}+a^{2} b_{1}^{19}+a b_{1}^{19}+a^{8} b_{1}^{16}+a^{4} b_{1}^{16}\right) k^{8}+\left(a^{2} b_{1}^{23}+a b_{1}^{23}+b_{1}^{23}\right. \\
& \left.+a^{4} b_{1}^{22}+a^{4} b_{1}^{21}+a^{2} b_{1}^{20}+a b_{1}^{20}+a^{2} b_{1}^{19}+a b_{1}^{19}+b_{1}^{19}+a^{16} b_{1}^{18}+b_{1}^{18}+a^{16} b_{1}^{17}\right) k^{7} \\
& +\left(b_{1}^{22}+a^{4} b_{1}^{21}+a^{3} b_{1}^{21}+a^{2} b_{1}^{21}+a b_{1}^{21}+a^{6} b_{1}^{20}+a^{5} b_{1}^{20}+a^{4} b_{1}^{20}+a^{2} b_{1}^{20}+a b_{1}^{20}+b_{1}^{20}\right. \\
& +a^{6} b_{1}^{19}+a^{4} b_{1}^{19}+a^{16} b_{1}^{18}+a^{4} b_{1}^{18}+a^{2} b_{1}^{18}+a b_{1}^{18}+a^{4} b_{1}^{17}+a^{3} b_{1}^{17}+a^{2} b_{1}^{17}+a b_{1}^{17} \\
& \left.+a^{18} b_{1}^{16}+a^{17} b_{1}^{16}+a^{16} b_{1}^{16}+a^{2} b_{1}^{16}+a^{18} b_{1}^{15}+a^{16} b_{1}^{15}\right) k^{6}+\left(b_{1}^{14} a^{20}+b_{1}^{13} a^{20}+b_{1}^{16} a^{17}\right. \\
& +b_{1}^{15} a^{17}+b_{1}^{13} a^{16}+b_{1}^{18} a^{8}+b_{1}^{17} a^{8}+b_{1}^{19} a^{6}+b_{1}^{16} a^{6}+b_{1}^{15} a^{6}+b_{1}^{20} a^{5}+b_{1}^{16} a^{5}+b_{1}^{15} a^{5} \\
& +b_{1}^{20} a^{4}+b_{1}^{19} a^{4}+b_{1}^{17} a^{4}+b_{1}^{15} a^{4}+b_{1}^{14} a^{4}+b_{1}^{20} a^{3}+b_{1}^{17} a^{3}+b_{1}^{18} a^{2}+b_{1}^{16} a^{2}+b_{1}^{19} a \\
& \left.+b_{1}^{18} a+b_{1}^{17} a+b_{1}^{20}+b_{1}^{18}\right) k^{5}+\left(b_{1}^{8} a^{32}+b_{1}^{12} a^{22}+b_{1}^{11} a^{22}+b_{1}^{12} a^{21}+b_{1}^{14} a^{20}+b_{1}^{12} a^{20}\right. \\
& +b_{1}^{11} a^{20}+b_{1}^{14} a^{19}+b_{1}^{13} a^{19}+b_{1}^{13} a^{18}+b_{1}^{11} a^{18}+b_{1}^{15} a^{17}+b_{1}^{13} a^{17}+b_{1}^{12} a^{17}+b_{1}^{16} a^{16} \\
& +b_{1}^{15} a^{16}+b_{1}^{13} a^{16}+b_{1}^{12} a^{16}+b_{1}^{11} a^{16}+b_{1}^{8} a^{16}+b_{1}^{16} a^{10}+b_{1}^{15} a^{10}+b_{1}^{16} a^{9}+b_{1}^{17} a^{8} \\
& +b_{1}^{15} a^{8}+b_{1}^{14} a^{8}+b_{1}^{13} a^{8}+b_{1}^{18} a^{7}+b_{1}^{13} a^{7}+b_{1}^{18} a^{6}+b_{1}^{16} a^{6}+b_{1}^{15} a^{6}+b_{1}^{14} a^{6}+b_{1}^{13} a^{6} \\
& +b_{1}^{12} a^{6}+b_{1}^{18} a^{5}+b_{1}^{15} a^{5}+b_{1}^{14} a^{5}+b_{1}^{13} a^{5}+b_{1}^{17} a^{4}+b_{1}^{14} a^{4}+b_{1}^{18} a^{3}+b_{1}^{16} a^{3}+b_{1}^{18} a^{2}
\end{aligned}
$$




$$
\begin{aligned}
& \left.+b_{1}^{16} a^{2}+b_{1}^{18} a\right) k^{4}+\left(b_{1}^{10} a^{24}+b_{1}^{9} a^{24}+b_{1}^{12} a^{18}+b_{1}^{11} a^{18}+b_{1}^{12} a^{17}+b_{1}^{11} a^{17}+b_{1}^{13} a^{16}\right. \\
& +b_{1}^{11} a^{16}+b_{1}^{9} a^{16}+b_{1}^{14} a^{12}+b_{1}^{13} a^{12}+b_{1}^{15} a^{10}+b_{1}^{12} a^{10}+b_{1}^{11} a^{10}+b_{1}^{15} a^{9}+b_{1}^{12} a^{9} \\
& +b_{1}^{11} a^{9}+b_{1}^{15} a^{8}+b_{1}^{11} a^{8}+b_{1}^{10} a^{8}+b_{1}^{16} a^{6}+b_{1}^{15} a^{6}+b_{1}^{16} a^{5}+b_{1}^{15} a^{5}+b_{1}^{16} a^{4}+b_{1}^{15} a^{4} \\
& \left.+b_{1}^{14} a^{4}+b_{1}^{17} a^{3}+b_{1}^{16} a^{3}+b_{1}^{17} a\right) k^{3}+\left(b_{1}^{4} a^{32}+b_{1}^{8} a^{26}+b_{1}^{7} a^{26}+b_{1}^{8} a^{25}+b_{1}^{10} a^{24}\right. \\
& +b_{1}^{8} a^{24}+b_{1}^{7} a^{24}+b_{1}^{10} a^{20}+b_{1}^{9} a^{20}+b_{1}^{9} a^{19}+b_{1}^{12} a^{18}+b_{1}^{11} a^{18}+b_{1}^{10} a^{18}+b_{1}^{9} a^{18}+b_{1}^{7} a^{18} \\
& +b_{1}^{10} a^{17}+b_{1}^{9} a^{17}+b_{1}^{8} a^{17}+b_{1}^{12} a^{16}+b_{1}^{11} a^{16}+b_{1}^{10} a^{16}+b_{1}^{8} a^{16}+b_{1}^{7} a^{16}+b_{1}^{4} a^{16}+b_{1}^{12} a^{14} \\
& +b_{1}^{11} a^{14}+b_{1}^{12} a^{13}+b_{1}^{13} a^{12}+b_{1}^{12} a^{12}+b_{1}^{11} a^{12}+b_{1}^{10} a^{12}+b_{1}^{9} a^{12}+b_{1}^{13} a^{11}+b_{1}^{9} a^{11} \\
& +b_{1}^{13} a^{10}+b_{1}^{12} a^{10}+b_{1}^{10} a^{10}+b_{1}^{9} a^{10}+b_{1}^{8} a^{10}+b_{1}^{13} a^{9}+b_{1}^{12} a^{9}+b_{1}^{10} a^{9}+b_{1}^{9} a^{9}+b_{1}^{13} a^{8} \\
& \left.+b_{1}^{13} a^{7}+b_{1}^{13} a^{6}+b_{1}^{12} a^{6}+b_{1}^{13} a^{5}\right) k^{2}+\left(b_{1}^{4} a^{32}+b_{1}^{6} a^{28}+b_{1}^{5} a^{28}+b_{1}^{8} a^{25}+b_{1}^{7} a^{25}+b_{1}^{5} a^{24}\right. \\
& +b_{1}^{8} a^{22}+b_{1}^{7} a^{22}+b_{1}^{8} a^{21}+b_{1}^{7} a^{21}+b_{1}^{9} a^{20}+b_{1}^{7} a^{20}+b_{1}^{5} a^{20}+b_{1}^{9} a^{19}+b_{1}^{10} a^{18}+b_{1}^{8} a^{18} \\
& +b_{1}^{10} a^{17}+b_{1}^{9} a^{17}+b_{1}^{8} a^{17}+b_{1}^{7} a^{17}+b_{1}^{5} a^{16}+b_{1}^{4} a^{16}+b_{1}^{11} a^{14}+b_{1}^{8} a^{14}+b_{1}^{7} a^{14}+b_{1}^{12} a^{13} \\
& +b_{1}^{8} a^{13}+b_{1}^{7} a^{13}+b_{1}^{12} a^{12}+b_{1}^{11} a^{12}+b_{1}^{9} a^{12}+b_{1}^{7} a^{12}+b_{1}^{6} a^{12}+b_{1}^{12} a^{11}+b_{1}^{9} a^{11}+b_{1}^{12} a^{10} \\
& \left.+b_{1}^{11} a^{10}+b_{1}^{10} a^{10}+b_{1}^{8} a^{10}+b_{1}^{12} a^{9}+b_{1}^{10} a^{9}+b_{1}^{9} a^{9}+b_{1}^{12} a^{8}+b_{1}^{11} a^{8}+b_{1}^{12} a^{7}+b_{1}^{12} a^{6}\right) k,
\end{aligned}
$$

$$
\begin{aligned}
& h_{2}=a^{20}+a^{18}+a^{17} b_{1}+a^{16} b_{1}^{4} k^{2}+a^{16} b_{1}^{2} k+a^{15} b_{1}^{3}+a^{13} b_{1}^{5} k+a^{12} b_{1}^{4}+a^{12}+a^{11} b_{1}^{7} k^{2} \\
& +a^{10} b_{1}^{4}+a^{10}+a^{9} b_{1}^{9} k^{3}+a^{9} b_{1}^{5}+a^{9} b_{1}+a^{8} b_{1}^{8} k^{2}+a^{8} b_{1}^{6} k+a^{8} b_{1}^{4} k^{2}+a^{8} b_{1}^{4}+a^{8} b_{1}^{2} k \\
& +a^{7} b_{1}^{11} k^{4}+a^{7} b_{1}^{7} k+a^{7} b_{1}^{3}+a^{6} b_{1}^{4}+a^{5} b_{1}^{13} k^{5}+a^{5} b_{1}^{9} k^{2}+a^{5} b_{1}^{5} k+a^{5} b_{1}^{5}+a^{4} b_{1}^{16} k^{8} \\
& +a^{4} b_{1}^{12} k^{4}+a^{4} b_{1}^{8} k^{4}+a^{4} b_{1}^{8} k^{2}+a^{4} b_{1}^{6} k+a^{3} b_{1}^{15} k^{6}+a^{3} b_{1}^{11} k^{3}+a^{3} b_{1}^{7} k^{2}+a^{3} b_{1}^{7} k \\
& +a^{2} b_{1}^{16} k^{8}+a^{2} b_{1}^{12} k^{4}+a^{2} b_{1}^{8} k^{4}+a b_{1}^{17} k^{8}+a b_{1}^{17} k^{7}+a b_{1}^{9} k^{4}+a b_{1}^{9} k^{3}+a b_{1}^{9} k^{2}+b_{1}^{20} k^{10} \\
& +b_{1}^{18} k^{9}+b_{1}^{16} k^{6}+b_{1}^{14} k^{5}+b_{1}^{12} k^{6}+b_{1}^{10} k^{5},
\end{aligned}
$$

$$
\begin{aligned}
& h_{3}=a^{50}+b_{1}^{2} a^{48}+a^{48}+b_{1}^{2} a^{44}+b_{1}^{4} a^{42}+b_{1}^{2} a^{42}+a^{42}+b_{1}^{6} a^{40}+a^{40}+b_{1}^{8} a^{38}+b_{1}^{6} a^{38} \\
& +b_{1}^{4} a^{38}+b_{1}^{2} a^{38}+b_{1}^{6} a^{36}+b_{1}^{6} a^{34}+a^{34}+b_{1}^{10} a^{32}+b_{1}^{8} a^{32}+b_{1}^{2} a^{32}+a^{32}+b_{1}^{10} a^{28} \\
& +b_{1}^{2} a^{28}+b_{1}^{12} a^{26}+b_{1}^{10} a^{26}+b_{1}^{4} a^{26}+b_{1}^{2} a^{26}+a^{26}+b_{1}^{10} a^{24}+b_{1}^{6} a^{24}+a^{24}+b_{1}^{12} a^{22} \\
& +b_{1}^{10} a^{22}+b_{1}^{8} a^{22}+b_{1}^{6} a^{22}+b_{1}^{4} a^{22}+b_{1}^{2} a^{22}+b_{1}^{10} a^{20}+b_{1}^{6} a^{20}+b_{1}^{12} a^{18}+b_{1}^{10} a^{18}+b_{1}^{6} a^{18} \\
& +b_{1}^{8} a^{16}+b_{1}^{12} a^{14}+b_{1}^{10} a^{14}+\left(b_{1}^{50}+a^{2} b_{1}^{48}+b_{1}^{48}\right) k^{24}+\left(a b_{1}^{45}+b_{1}^{45}+a^{2} b_{1}^{44}\right) k^{21}+\left(a^{2} b_{1}^{44}\right. \\
& \left.+b_{1}^{44}+b_{1}^{43}+a^{4} b_{1}^{42}+a^{2} b_{1}^{42}+b_{1}^{42}+a^{2} b_{1}^{40}+b_{1}^{40}\right) k^{20}+\left(a b_{1}^{43}+a^{2} b_{1}^{42}+a b_{1}^{42}+b_{1}^{42}\right. \\
& \left.+a^{2} b_{1}^{41}+a b_{1}^{41}+a^{2} b_{1}^{40}\right) k^{19}+\left(a^{2} b_{1}^{44}+a b_{1}^{43}+a^{4} b_{1}^{42}+a b_{1}^{42}+b_{1}^{42}+a^{4} b_{1}^{40}+a^{2} b_{1}^{40}\right. \\
& \left.+a^{4} b_{1}^{39}+a^{4} b_{1}^{38}+a^{2} b_{1}^{38}\right) k^{18}+\left(a b_{1}^{41}+b_{1}^{41}+a^{4} b_{1}^{40}+a^{3} b_{1}^{40}+a^{2} b_{1}^{40}+a^{5} b_{1}^{39}+a^{3} b_{1}^{39}\right. \\
& \left.+a^{6} b_{1}^{38}+a^{5} b_{1}^{38}+a^{9} b_{1}^{37}+a^{8} b_{1}^{37}+a^{6} b_{1}^{37}+a^{5} b_{1}^{37}+a^{10} b_{1}^{36}+a^{6} b_{1}^{36}\right) k^{17}+\left(b_{1}^{42}+a^{6} b_{1}^{40}\right. \\
& +b_{1}^{40}+b_{1}^{39}+a^{8} b_{1}^{38}+a^{6} b_{1}^{38}+a^{4} b_{1}^{38}+a^{2} b_{1}^{38}+b_{1}^{38}+a^{10} b_{1}^{36}+a^{6} b_{1}^{36}+a^{16} b_{1}^{34}+a^{12} b_{1}^{34} \\
& \left.+a^{10} b_{1}^{34}+a^{6} b_{1}^{34}+b_{1}^{34}+a^{18} b_{1}^{32}+a^{16} b_{1}^{32}+a^{10} b_{1}^{32}+a^{8} b_{1}^{32}+a^{2} b_{1}^{32}+b_{1}^{32}\right) k^{16}+\left(a b_{1}^{39}\right. \\
& \left.+a^{2} b_{1}^{38}+a^{2} b_{1}^{37}+a b_{1}^{37}+a^{2} b_{1}^{36}\right) k^{15}+\left(a^{3} b_{1}^{37}+a^{3} b_{1}^{36}+a^{2} b_{1}^{36}+b_{1}^{36}+a^{4} b_{1}^{35}+a^{4} b_{1}^{34}\right) k^{14} \\
& +\left(b_{1}^{36}+a^{6} b_{1}^{34}+a^{5} b_{1}^{34}+a b_{1}^{34}+b_{1}^{34}+a^{6} b_{1}^{33}+a^{5} b_{1}^{33}+a^{6} b_{1}^{32}+a b_{1}^{29}+b_{1}^{29}+a^{2} b_{1}^{28}\right) k^{13} \\
& +\left(a^{4} b_{1}^{34}+a^{2} b_{1}^{34}+a^{3} b_{1}^{33}+a^{3} b_{1}^{32}+a^{2} b_{1}^{32}+a^{8} b_{1}^{31}+a^{8} b_{1}^{30}+a^{2} b_{1}^{28}+b_{1}^{28}+b_{1}^{27}+a^{4} b_{1}^{26}\right.
\end{aligned}
$$




$$
\begin{aligned}
& \left.+a^{2} b_{1}^{26}+b_{1}^{26}+a^{2} b_{1}^{24}+b_{1}^{24}\right) k^{12}+\left(a^{3} b_{1}^{33}+a^{9} b_{1}^{31}+a^{5} b_{1}^{31}+a^{3} b_{1}^{31}+a^{10} b_{1}^{30}+a^{5} b_{1}^{30}\right. \\
& +a^{4} b_{1}^{30}+a b_{1}^{30}+b_{1}^{30}+a^{10} b_{1}^{29}+a^{9} b_{1}^{29}+a^{10} b_{1}^{28}+a b_{1}^{27}+a^{2} b_{1}^{26}+a b_{1}^{26}+b_{1}^{26}+a^{2} b_{1}^{25} \\
& \left.+a b_{1}^{25}+a^{2} b_{1}^{24}\right) k^{11}+\left(a^{6} b_{1}^{32}+a^{5} b_{1}^{31}+a b_{1}^{31}+a^{5} b_{1}^{30}+a^{2} b_{1}^{30}+a b_{1}^{30}+a^{11} b_{1}^{29}+a^{7} b_{1}^{29}\right. \\
& +a^{3} b_{1}^{29}+a^{11} b_{1}^{28}+a^{10} b_{1}^{28}+a^{8} b_{1}^{28}+a^{7} b_{1}^{28}+a^{4} b_{1}^{28}+a^{3} b_{1}^{28}+a^{2} b_{1}^{28}+b_{1}^{28}+a^{12} b_{1}^{27} \\
& \left.+a b_{1}^{27}+a^{12} b_{1}^{26}+a^{4} b_{1}^{26}+a b_{1}^{26}+b_{1}^{26}+a^{4} b_{1}^{24}+a^{2} b_{1}^{24}+a^{4} b_{1}^{23}+a^{4} b_{1}^{22}+a^{2} b_{1}^{22}\right) k^{10} \\
& +\left(a^{5} b_{1}^{29}+a^{4} b_{1}^{29}+a b_{1}^{29}+b_{1}^{29}+a^{8} b_{1}^{28}+a^{7} b_{1}^{28}+a^{6} b_{1}^{28}+a^{4} b_{1}^{28}+a^{3} b_{1}^{28}+a^{2} b_{1}^{28}+b_{1}^{28}\right. \\
& +a^{9} b_{1}^{27}+a^{7} b_{1}^{27}+a^{5} b_{1}^{27}+a^{3} b_{1}^{27}+a^{14} b_{1}^{26}+a^{13} b_{1}^{26}+a b_{1}^{26}+b_{1}^{26}+a^{14} b_{1}^{25}+a^{13} b_{1}^{25} \\
& +a b_{1}^{25}+b_{1}^{25}+a^{14} b_{1}^{24}+a^{4} b_{1}^{24}+a^{3} b_{1}^{24}+a^{2} b_{1}^{24}+a^{5} b_{1}^{23}+a^{3} b_{1}^{23}+a^{6} b_{1}^{22}+a^{5} b_{1}^{22} \\
& \left.+a^{9} b_{1}^{21}+a^{8} b_{1}^{21}+a^{6} b_{1}^{21}+a^{5} b_{1}^{21}+a^{10} b_{1}^{20}+a^{6} b_{1}^{20}\right) k^{9}+\left(b_{1}^{16} a^{34}+b_{1}^{18} a^{32}+b_{1}^{16} a^{32}\right. \\
& +b_{1}^{23} a^{16}+b_{1}^{22} a^{16}+b_{1}^{26} a^{12}+b_{1}^{18} a^{12}+b_{1}^{28} a^{10}+b_{1}^{26} a^{10}+b_{1}^{20} a^{10}+b_{1}^{18} a^{10}+b_{1}^{16} a^{10} \\
& +b_{1}^{26} a^{8}+b_{1}^{22} a^{8}+b_{1}^{16} a^{8}+b_{1}^{28} a^{6}+b_{1}^{26} a^{6}+b_{1}^{24} a^{6}+b_{1}^{22} a^{6}+b_{1}^{20} a^{6}+b_{1}^{18} a^{6}+b_{1}^{27} a^{4} \\
& \left.+b_{1}^{26} a^{4}+b_{1}^{22} a^{4}+b_{1}^{25} a^{3}+b_{1}^{24} a^{3}+b_{1}^{24} a^{2}+b_{1}^{22} a^{2}+b_{1}^{27}+b_{1}^{26}+b_{1}^{24}+b_{1}^{23}+b_{1}^{22}\right) k^{8} \\
& +\left(a^{5} b_{1}^{27}+a b_{1}^{27}+a^{6} b_{1}^{26}+a^{2} b_{1}^{26}+a^{6} b_{1}^{25}+a^{5} b_{1}^{25}+a^{3} b_{1}^{25}+a^{2} b_{1}^{25}+a b_{1}^{25}+a^{6} b_{1}^{24}\right. \\
& +a^{2} b_{1}^{24}+a^{17} b_{1}^{23}+a^{5} b_{1}^{23}+a^{3} b_{1}^{23}+a b_{1}^{23}+a^{18} b_{1}^{22}+a^{5} b_{1}^{22}+a^{4} b_{1}^{22}+a^{2} b_{1}^{22}+a b_{1}^{22} \\
& \left.+b_{1}^{22}+a^{18} b_{1}^{21}+a^{17} b_{1}^{21}+a^{2} b_{1}^{21}+a b_{1}^{21}+a^{18} b_{1}^{20}+a^{2} b_{1}^{20}\right) k^{7}+\left(a^{4} b_{1}^{26}+b_{1}^{26}+a^{7} b_{1}^{25}\right. \\
& +a^{3} b_{1}^{25}+a^{7} b_{1}^{24}+a^{6} b_{1}^{24}+a^{4} b_{1}^{24}+a^{3} b_{1}^{24}+a^{2} b_{1}^{24}+b_{1}^{24}+a^{8} b_{1}^{23}+a^{5} b_{1}^{23}+a^{4} b_{1}^{23}+a b_{1}^{23} \\
& +a^{8} b_{1}^{22}+a^{5} b_{1}^{22}+a^{4} b_{1}^{22}+a b_{1}^{22}+a^{19} b_{1}^{21}+a^{7} b_{1}^{21}+a^{19} b_{1}^{20}+a^{18} b_{1}^{20}+a^{16} b_{1}^{20}+a^{7} b_{1}^{20} \\
& \left.+a^{4} b_{1}^{20}+a^{2} b_{1}^{20}+a^{20} b_{1}^{19}+a^{4} b_{1}^{19}+a^{20} b_{1}^{18}+a^{4} b_{1}^{18}\right) k^{6}+\left(b_{1}^{12} a^{34}+b_{1}^{13} a^{33}+b_{1}^{13} a^{32}\right. \\
& +b_{1}^{18} a^{22}+b_{1}^{17} a^{22}+b_{1}^{16} a^{22}+b_{1}^{18} a^{21}+b_{1}^{17} a^{21}+b_{1}^{12} a^{18}+b_{1}^{18} a^{17}+b_{1}^{13} a^{17}+b_{1}^{20} a^{16} \\
& +b_{1}^{18} a^{16}+b_{1}^{13} a^{16}+b_{1}^{22} a^{10}+b_{1}^{21} a^{10}+b_{1}^{22} a^{9}+b_{1}^{19} a^{9}+b_{1}^{18} a^{9}+b_{1}^{21} a^{8}+b_{1}^{18} a^{8}+b_{1}^{20} a^{7} \\
& +b_{1}^{19} a^{7}+b_{1}^{22} a^{6}+b_{1}^{21} a^{6}+b_{1}^{18} a^{6}+b_{1}^{17} a^{6}+b_{1}^{16} a^{6}+b_{1}^{19} a^{5}+b_{1}^{18} a^{5}+b_{1}^{17} a^{5}+b_{1}^{24} a^{4} \\
& \left.+b_{1}^{22} a^{4}+b_{1}^{21} a^{4}+b_{1}^{20} a^{4}+b_{1}^{20} a^{3}+b_{1}^{19} a^{3}+b_{1}^{22} a+b_{1}^{24}+b_{1}^{22}\right) k^{5}+\left(b_{1}^{10} a^{36}+b_{1}^{12} a^{34}\right. \\
& +b_{1}^{10} a^{34}+b_{1}^{8} a^{34}+b_{1}^{12} a^{32}+b_{1}^{11} a^{32}+b_{1}^{10} a^{32}+b_{1}^{8} a^{32}+b_{1}^{15} a^{24}+b_{1}^{14} a^{24}+b_{1}^{18} a^{20} \\
& +b_{1}^{10} a^{20}+b_{1}^{17} a^{19}+b_{1}^{16} a^{19}+b_{1}^{18} a^{18}+b_{1}^{16} a^{18}+b_{1}^{12} a^{18}+b_{1}^{10} a^{18}+b_{1}^{8} a^{18}+b_{1}^{12} a^{16} \\
& +b_{1}^{11} a^{16}+b_{1}^{10} a^{16}+b_{1}^{8} a^{16}+b_{1}^{19} a^{12}+b_{1}^{17} a^{11}+b_{1}^{16} a^{11}+b_{1}^{20} a^{10}+b_{1}^{18} a^{10}+b_{1}^{16} a^{10} \\
& +b_{1}^{22} a^{8}+b_{1}^{20} a^{8}+b_{1}^{15} a^{8}+b_{1}^{14} a^{8}+b_{1}^{21} a^{7}+b_{1}^{20} a^{7}+b_{1}^{22} a^{6}+b_{1}^{22} a^{4}+b_{1}^{20} a^{4}+b_{1}^{19} a^{4} \\
& \left.+b_{1}^{18} a^{4}+b_{1}^{21} a^{3}+b_{1}^{20} a^{3}+b_{1}^{22} a^{2}+b_{1}^{20} a^{2}\right) k^{4}+\left(b_{1}^{10} a^{34}+b_{1}^{9} a^{34}+b_{1}^{8} a^{34}+b_{1}^{11} a^{33}\right. \\
& +b_{1}^{10} a^{33}+b_{1}^{9} a^{33}+b_{1}^{10} a^{32}+b_{1}^{14} a^{26}+b_{1}^{13} a^{26}+b_{1}^{12} a^{26}+b_{1}^{15} a^{25}+b_{1}^{13} a^{25}+b_{1}^{15} a^{21} \\
& +b_{1}^{14} a^{21}+b_{1}^{14} a^{20}+b_{1}^{17} a^{19}+b_{1}^{15} a^{19}+b_{1}^{10} a^{18}+b_{1}^{9} a^{18}+b_{1}^{8} a^{18}+b_{1}^{14} a^{17}+b_{1}^{11} a^{17} \\
& +b_{1}^{10} a^{17}+b_{1}^{9} a^{17}+b_{1}^{14} a^{16}+b_{1}^{10} a^{16}+b_{1}^{18} a^{14}+b_{1}^{17} a^{14}+b_{1}^{16} a^{14}+b_{1}^{19} a^{13}+b_{1}^{17} a^{13} \\
& +b_{1}^{15} a^{13}+b_{1}^{14} a^{13}+b_{1}^{14} a^{12}+b_{1}^{17} a^{11}+b_{1}^{15} a^{11}+b_{1}^{14} a^{10}+b_{1}^{13} a^{10}+b_{1}^{12} a^{10}+b_{1}^{19} a^{9} \\
& +b_{1}^{15} a^{9}+b_{1}^{14} a^{9}+b_{1}^{13} a^{9}+b_{1}^{14} a^{8}+b_{1}^{21} a^{7}+b_{1}^{19} a^{7}+b_{1}^{18} a^{6}+b_{1}^{17} a^{6}+b_{1}^{16} a^{6}+b_{1}^{17} a^{5} \\
& \left.+b_{1}^{21} a^{3}+b_{1}^{19} a^{3}\right) k^{3}+\left(b_{1}^{10} a^{36}+b_{1}^{8} a^{36}+b_{1}^{7} a^{36}+b_{1}^{6} a^{36}+b_{1}^{12} a^{34}+b_{1}^{8} a^{34}+b_{1}^{6} a^{34}\right. \\
& +b_{1}^{11} a^{33}+b_{1}^{10} a^{33}+b_{1}^{10} a^{32}+b_{1}^{11} a^{28}+b_{1}^{10} a^{28}+b_{1}^{13} a^{27}+b_{1}^{12} a^{27}+b_{1}^{12} a^{26}+b_{1}^{12} a^{24} \\
& +b_{1}^{13} a^{23}+b_{1}^{12} a^{23}+b_{1}^{16} a^{22}+b_{1}^{15} a^{21}+b_{1}^{14} a^{21}+b_{1}^{12} a^{20}+b_{1}^{10} a^{20}+b_{1}^{8} a^{20}+b_{1}^{7} a^{20}
\end{aligned}
$$




$$
\begin{aligned}
& +b_{1}^{6} a^{20}+b_{1}^{13} a^{19}+b_{1}^{12} a^{19}+b_{1}^{14} a^{18}+b_{1}^{12} a^{18}+b_{1}^{8} a^{18}+b_{1}^{6} a^{18}+b_{1}^{15} a^{17}+b_{1}^{14} a^{17} \\
& +b_{1}^{11} a^{17}+b_{1}^{10} a^{17}+b_{1}^{15} a^{16}+b_{1}^{14} a^{16}+b_{1}^{12} a^{16}+b_{1}^{10} a^{16}+b_{1}^{17} a^{15}+b_{1}^{16} a^{15}+b_{1}^{13} a^{15} \\
& +b_{1}^{12} a^{15}+b_{1}^{16} a^{14}+b_{1}^{15} a^{13}+b_{1}^{14} a^{13}+b_{1}^{12} a^{12}+b_{1}^{11} a^{12}+b_{1}^{10} a^{12}+b_{1}^{14} a^{10}+b_{1}^{12} a^{10} \\
& \left.+b_{1}^{15} a^{9}+b_{1}^{14} a^{9}+b_{1}^{15} a^{8}+b_{1}^{14} a^{8}+b_{1}^{17} a^{7}+b_{1}^{16} a^{7}\right) k^{2}+\left(b_{1}^{4} a^{42}+b_{1}^{5} a^{41}+b_{1}^{5} a^{40}+b_{1}^{6} a^{38}\right. \\
& +b_{1}^{5} a^{38}+b_{1}^{4} a^{38}+b_{1}^{7} a^{37}+b_{1}^{6} a^{37}+b_{1}^{5} a^{37}+b_{1}^{8} a^{36}+b_{1}^{8} a^{35}+b_{1}^{7} a^{35}+b_{1}^{8} a^{34}+b_{1}^{9} a^{33} \\
& +b_{1}^{9} a^{32}+b_{1}^{10} a^{30}+b_{1}^{9} a^{30}+b_{1}^{8} a^{30}+b_{1}^{10} a^{29}+b_{1}^{9} a^{29}+b_{1}^{4} a^{26}+b_{1}^{11} a^{25}+b_{1}^{5} a^{25}+b_{1}^{12} a^{24} \\
& +b_{1}^{5} a^{24}+b_{1}^{12} a^{23}+b_{1}^{11} a^{23}+b_{1}^{12} a^{22}+b_{1}^{6} a^{22}+b_{1}^{5} a^{22}+b_{1}^{4} a^{22}+b_{1}^{13} a^{21}+b_{1}^{11} a^{21}+b_{1}^{7} a^{21} \\
& +b_{1}^{6} a^{21}+b_{1}^{5} a^{21}+b_{1}^{13} a^{20}+b_{1}^{12} a^{20}+b_{1}^{8} a^{20}+b_{1}^{12} a^{19}+b_{1}^{11} a^{19}+b_{1}^{8} a^{19}+b_{1}^{7} a^{19}+b_{1}^{14} a^{18} \\
& +b_{1}^{13} a^{18}+b_{1}^{12} a^{18}+b_{1}^{8} a^{18}+b_{1}^{14} a^{17}+b_{1}^{13} a^{17}+b_{1}^{11} a^{17}+b_{1}^{9} a^{17}+b_{1}^{12} a^{16}+b_{1}^{9} a^{16}+b_{1}^{12} a^{15} \\
& +b_{1}^{11} a^{15}+b_{1}^{12} a^{14}+b_{1}^{10} a^{14}+b_{1}^{9} a^{14}+b_{1}^{8} a^{14}+b_{1}^{13} a^{13}+b_{1}^{11} a^{13}+b_{1}^{10} a^{13}+b_{1}^{9} a^{13}+b_{1}^{13} a^{12} \\
& \left.+b_{1}^{12} a^{12}+b_{1}^{12} a^{11}+b_{1}^{11} a^{11}+b_{1}^{14} a^{10}+b_{1}^{13} a^{10}+b_{1}^{12} a^{10}+b_{1}^{14} a^{9}+b_{1}^{13} a^{9}\right) k .
\end{aligned}
$$

In (3.36),

(A4)

$$
\begin{aligned}
& h_{1}^{\prime}=a^{22}+b_{1}^{4} a^{20}+b_{1}^{2} a^{20}+a^{20}+b_{1} a^{19}+b_{1}^{5} a^{17}+b_{1}^{3} a^{17}+b_{1}^{4} a^{16}+b_{1}^{6} a^{14}+a^{14}+b_{1}^{6} a^{12} \\
& +b_{1}^{2} a^{12}+a^{12}+b_{1} a^{11}+b_{1}^{4} a^{10}+b_{1}^{7} a^{9}+b_{1}^{3} a^{9}+b_{1}^{6} a^{8}+b_{1}^{6} a^{6}+b_{1}^{4} a^{6}+b_{1}^{7} a^{5}+b_{1}^{5} a^{5} \\
& +\left(a^{3} b_{1}^{19}+a^{4} b_{1}^{18}+a^{2} b_{1}^{18}\right) k^{9}+\left(a^{4} b_{1}^{20}+a^{2} b_{1}^{20}+b_{1}^{20}+a^{4} b_{1}^{18}+a^{3} b_{1}^{17}+a^{6} b_{1}^{16}+a^{4} b_{1}^{16}\right) k^{8} \\
& +\left(a^{3} b_{1}^{19}+a^{4} b_{1}^{18}+a^{2} b_{1}^{18}+b_{1}^{18}+a^{3} b_{1}^{17}+a b_{1}^{17}\right) k^{7}+\left(b_{1}^{18}+a^{5} b_{1}^{17}+a b_{1}^{17}+a^{6} b_{1}^{16}+a^{4} b_{1}^{16}\right. \\
& \left.+a^{2} b_{1}^{16}+b_{1}^{16}+a^{5} b_{1}^{15}+a b_{1}^{15}\right) k^{6}+\left(b_{1}^{16}+a^{7} b_{1}^{15}+a^{8} b_{1}^{14}+b_{1}^{14}+a^{7} b_{1}^{13}+a^{5} b_{1}^{13}+a^{3} b_{1}^{13}\right. \\
& \left.+a b_{1}^{13}+a^{3} b_{1}^{11}+a^{4} b_{1}^{10}+a^{2} b_{1}^{10}\right) k^{5}+\left(a^{6} b_{1}^{14}+a^{2} b_{1}^{14}+a^{9} b_{1}^{13}+a b_{1}^{13}+a^{10} b_{1}^{12}+a^{4} b_{1}^{12}\right. \\
& \left.+a^{9} b_{1}^{11}+a b_{1}^{11}+a^{4} b_{1}^{10}+a^{3} b_{1}^{9}+a^{6} b_{1}^{8}+a^{4} b_{1}^{8}\right) k^{4}+\left(a^{3} b_{1}^{13}+a^{6} b_{1}^{12}+a^{4} b_{1}^{12}+a^{2} b_{1}^{12}\right. \\
& \left.+a^{11} b_{1}^{11}+a^{3} b_{1}^{11}+a b_{1}^{11}+a^{12} b_{1}^{10}+a^{10} b_{1}^{10}+a^{8} b_{1}^{10}+a^{6} b_{1}^{10}+a^{11} b_{1}^{9}+a^{9} b_{1}^{9}\right) k^{3}+\left(b_{1}^{8} a^{14}\right. \\
& \left.+b_{1}^{9} a^{13}+b_{1}^{7} a^{13}+b_{1}^{8} a^{12}+b_{1}^{8} a^{10}+b_{1}^{9} a^{9}+b_{1}^{7} a^{9}+b_{1}^{11} a^{5}+b_{1}^{9} a^{5}+b_{1}^{8} a^{4}+b_{1}^{11} a+b_{1}^{9} a\right) k^{2} \\
& +\left(b_{1}^{2} a^{20}+b_{1}^{3} a^{19}+b_{1}^{2} a^{18}+b_{1}^{6} a^{16}+b_{1}^{7} a^{15}+b_{1}^{5} a^{15}+b_{1}^{5} a^{13}+b_{1}^{2} a^{12}+b_{1}^{5} a^{11}+b_{1}^{3} a^{11}\right. \\
& +b_{1}^{8} a^{10}+b_{1}^{6} a^{10}+b_{1}^{2} a^{10}+b_{1}^{5} a^{9}+b_{1}^{8} a^{8}+b_{1}^{9} a^{7}+b_{1}^{8} a^{6}+b_{1}^{6} a^{6}+b_{1}^{8} a^{4}+b_{1}^{6} a^{4}+b_{1}^{9} a^{3} \\
& \left.+b_{1}^{7} a^{3}\right) k,
\end{aligned}
$$

(A5)

$$
\begin{aligned}
& h_{3}^{\prime}=b_{1}^{2} a^{24}+a^{24}+b_{1}^{3} a^{23}+b_{1}^{4} a^{22}+b_{1}^{2} a^{22}+b_{1}^{5} a^{21}+b_{1}^{4} a^{20}+b_{1}^{2} a^{20}+a^{20}+b_{1}^{5} a^{19} \\
& +b_{1}^{3} a^{19}+b_{1}^{4} a^{18}+b_{1}^{7} a^{17}+b_{1}^{5} a^{17}+b_{1}^{8} a^{16}+b_{1}^{6} a^{16}+b_{1}^{2} a^{16}+a^{16}+b_{1}^{5} a^{15}+b_{1}^{3} a^{15}+b_{1}^{8} a^{14} \\
& +b_{1}^{6} a^{14}+b_{1}^{4} a^{14}+b_{1}^{2} a^{14}+b_{1}^{9} a^{13}+b_{1}^{5} a^{13}+b_{1}^{8} a^{12}+b_{1}^{4} a^{12}+b_{1}^{2} a^{12}+a^{12}+b_{1}^{7} a^{11}+b_{1}^{5} a^{11} \\
& +b_{1}^{3} a^{11}+b_{1}^{10} a^{10}+b_{1}^{6} a^{10}+b_{1}^{4} a^{10}+b_{1}^{5} a^{9}+b_{1}^{6} a^{8}+b_{1}^{7} a^{7}+b_{1}^{5} a^{7}+b_{1}^{10} a^{6}+b_{1}^{8} a^{6}+b_{1}^{9} a^{5} \\
& +b_{1}^{7} a^{5}+\left(a^{4} b_{1}^{24}+a^{3} b_{1}^{23}+a^{4} b_{1}^{22}+a^{5} b_{1}^{21}+a^{3} b_{1}^{21}+a^{4} b_{1}^{20}+a^{5} b_{1}^{19}+a b_{1}^{19}+a^{6} b_{1}^{18}\right. \\
& \left.+a^{2} b_{1}^{18}\right) k^{9}+\left(a^{3} b_{1}^{23}+a^{6} b_{1}^{22}+a^{2} b_{1}^{22}+b_{1}^{22}+a^{5} b_{1}^{21}+a^{3} b_{1}^{21}+a b_{1}^{21}+a^{2} b_{1}^{20}+a^{5} b_{1}^{19}\right. \\
& \left.+a^{3} b_{1}^{19}+a^{8} b_{1}^{18}+a^{6} b_{1}^{18}+a^{2} b_{1}^{18}+a^{8} b_{1}^{16}+a^{4} b_{1}^{16}\right) k^{8}+\left(a^{2} b_{1}^{22}+a b_{1}^{21}+a^{8} b_{1}^{20}+b_{1}^{20}\right. \\
& +a^{7} b_{1}^{19}+a^{5} b_{1}^{19}+a^{3} b_{1}^{19}+a b_{1}^{19}+a^{8} b_{1}^{18}+a^{4} b_{1}^{18}+a^{2} b_{1}^{18}+a^{9} b_{1}^{17}+a^{5} b_{1}^{17}+a^{6} b_{1}^{16} \\
& \left.+a^{4} b_{1}^{16}\right) k^{7}+\left(a b_{1}^{21}+a^{4} b_{1}^{20}+a^{2} b_{1}^{20}+b_{1}^{20}+a^{7} b_{1}^{19}+a^{10} b_{1}^{18}+a^{6} b_{1}^{18}+a^{4} b_{1}^{18}+b_{1}^{18}\right.
\end{aligned}
$$




$$
\begin{aligned}
& \left.+a^{7} b_{1}^{17}+a^{3} b_{1}^{17}+a^{10} b_{1}^{16}+a^{6} b_{1}^{16}+a^{11} b_{1}^{15}+a^{9} b_{1}^{15}+a^{7} b_{1}^{15}+a^{3} b_{1}^{15}+a^{8} b_{1}^{14}+a^{6} b_{1}^{14}\right) k^{6} \\
& +\left(a b_{1}^{19}+a^{2} b_{1}^{18}+b_{1}^{18}+a^{7} b_{1}^{17}+a^{5} b_{1}^{17}+a^{12} b_{1}^{16}+a^{8} b_{1}^{16}+a^{6} b_{1}^{16}+a^{4} b_{1}^{16}+a^{2} b_{1}^{16}+b_{1}^{16}\right. \\
& +a^{11} b_{1}^{15}+a^{7} b_{1}^{15}+a^{3} b_{1}^{15}+a b_{1}^{15}+a^{12} b_{1}^{14}+a^{10} b_{1}^{14}+a^{6} b_{1}^{14}+a^{4} b_{1}^{14}+a^{2} b_{1}^{14}+a^{13} b_{1}^{13} \\
& \left.+a^{7} b_{1}^{13}+a^{10} b_{1}^{12}+a^{8} b_{1}^{12}+a^{4} b_{1}^{12}+a^{5} b_{1}^{11}+a b_{1}^{11}+a^{6} b_{1}^{10}+a^{2} b_{1}^{10}\right) k^{5}+\left(a^{5} b_{1}^{17}+a^{3} b_{1}^{17}\right. \\
& +a^{6} b_{1}^{16}+a^{4} b_{1}^{16}+a^{2} b_{1}^{16}+a^{11} b_{1}^{15}+a^{9} b_{1}^{15}+a^{7} b_{1}^{15}+a b_{1}^{15}+a^{14} b_{1}^{14}+a^{10} b_{1}^{14}+a^{8} b_{1}^{14} \\
& +a^{6} b_{1}^{14}+a^{2} b_{1}^{14}+a^{9} b_{1}^{13}+a^{3} b_{1}^{13}+a b_{1}^{13}+a^{14} b_{1}^{12}+a^{12} b_{1}^{12}+a^{8} b_{1}^{12}+a^{2} b_{1}^{12}+a^{15} b_{1}^{11} \\
& \left.+a^{13} b_{1}^{11}+a^{7} b_{1}^{11}+a^{5} b_{1}^{11}+a^{12} b_{1}^{10}+a^{10} b_{1}^{10}+a^{8} b_{1}^{10}+a^{6} b_{1}^{10}+a^{2} b_{1}^{10}+a^{8} b_{1}^{8}+a^{4} b_{1}^{8}\right) k^{4} \\
& +\left(b_{1}^{9} a^{17}+b_{1}^{12} a^{16}+b_{1}^{10} a^{16}+b_{1}^{11} a^{15}+b_{1}^{8} a^{14}+b_{1}^{11} a^{13}+b_{1}^{9} a^{13}+b_{1}^{10} a^{12}+b_{1}^{8} a^{12}+b_{1}^{12} a^{10}\right. \\
& +b_{1}^{13} a^{9}+b_{1}^{11} a^{9}+b_{1}^{10} a^{8}+b_{1}^{13} a^{7}+b_{1}^{9} a^{7}+b_{1}^{10} a^{6}+b_{1}^{8} a^{6}+b_{1}^{15} a^{5}+b_{1}^{16} a^{4}+b_{1}^{10} a^{4}+b_{1}^{8} a^{4} \\
& \left.+b_{1}^{15} a^{3}+b_{1}^{13} a^{3}+b_{1}^{9} a^{3}+b_{1}^{14} a^{2}+b_{1}^{12} a^{2}+b_{1}^{10} a^{2}+b_{1}^{13} a+b_{1}^{11} a\right) k^{3}+\left(b_{1}^{7} a^{19}+b_{1}^{10} a^{18}\right. \\
& +b_{1}^{8} a^{18}+b_{1}^{7} a^{17}+b_{1}^{6} a^{16}+b_{1}^{11} a^{15}+b_{1}^{9} a^{15}+b_{1}^{7} a^{15}+b_{1}^{10} a^{14}+b_{1}^{8} a^{14}+b_{1}^{6} a^{14}+b_{1}^{9} a^{13} \\
& +b_{1}^{8} a^{12}+b_{1}^{11} a^{11}+b_{1}^{7} a^{11}+b_{1}^{12} a^{10}+b_{1}^{10} a^{10}+b_{1}^{8} a^{10}+b_{1}^{13} a^{9}+b_{1}^{11} a^{9}+b_{1}^{9} a^{9}+b_{1}^{7} a^{9} \\
& +b_{1}^{12} a^{8}+b_{1}^{6} a^{8}+b_{1}^{13} a^{7}+b_{1}^{11} a^{7}+b_{1}^{9} a^{7}+b_{1}^{7} a^{7}+b_{1}^{14} a^{6}+b_{1}^{12} a^{6}+b_{1}^{8} a^{6}+b_{1}^{6} a^{6}+b_{1}^{12} a^{4} \\
& \left.+b_{1}^{8} a^{4}+b_{1}^{13} a^{3}+b_{1}^{11} a^{3}+b_{1}^{14} a^{2}+b_{1}^{10} a^{2}+b_{1}^{13} a+b_{1}^{11} a\right) k^{2}+\left(b_{1}^{2} a^{22}+b_{1}^{3} a^{21}+b_{1}^{4} a^{20}\right. \\
& +b_{1}^{5} a^{19}+b_{1}^{6} a^{18}+b_{1}^{4} a^{18}+b_{1}^{2} a^{18}+b_{1}^{3} a^{17}+b_{1}^{8} a^{16}+b_{1}^{4} a^{16}+b_{1}^{9} a^{15}+b_{1}^{5} a^{15}+b_{1}^{10} a^{14} \\
& +b_{1}^{2} a^{14}+b_{1}^{9} a^{13}+b_{1}^{3} a^{13}+b_{1}^{8} a^{12}+b_{1}^{4} a^{12}+b_{1}^{7} a^{11}+b_{1}^{5} a^{11}+b_{1}^{8} a^{10}+b_{1}^{6} a^{10}+b_{1}^{4} a^{10} \\
& +b_{1}^{2} a^{10}+b_{1}^{7} a^{9}+b_{1}^{3} a^{9}+b_{1}^{12} a^{8}+b_{1}^{10} a^{8}+b_{1}^{4} a^{8}+b_{1}^{11} a^{7}+b_{1}^{7} a^{7}+b_{1}^{5} a^{7}+b_{1}^{10} a^{6}+b_{1}^{8} a^{6} \\
& \left.+b_{1}^{9} a^{5}+b_{1}^{7} a^{5}+b_{1}^{12} a^{4}+b_{1}^{10} a^{4}+b_{1}^{11} a^{3}+b_{1}^{9} a^{3}\right) k .
\end{aligned}
$$

In (3.37) - 3.40),

$$
\begin{aligned}
S_{1}= & b_{1}^{8} k^{3}+b_{1}^{4} k+b_{1}^{3}+b_{1}^{2} k+b_{1}^{2}+b_{1}+1, \\
S_{2}= & b_{1}^{16} k^{15}+b_{1}^{16} k^{14}+b_{1}^{16} k^{13}+b_{1}^{16} k^{12}+b_{1}^{14} k^{12}+b_{1}^{14} k^{10}+b_{1}^{12} k^{12} \\
& +b_{1}^{10} k^{12}+b_{1}^{10} k^{11}+b_{1}^{10} k^{10}+b_{1}^{10} k^{8}+b_{1}^{10} k^{6}+b_{1}^{8} k^{12}+b_{1}^{8} k^{11} \\
& +b_{1}^{8} k^{10}+b_{1}^{8} k^{9}+b_{1}^{8} k^{5}+b_{1}^{6} k^{7}+b_{1}^{6} k^{5}+b_{1}^{6} k^{4}+b_{1}^{4} k^{4}+b_{1}^{4} k^{3} \\
& +b_{1}^{2} k^{4}+b_{1}^{2} k^{3}+b_{1}^{2} k^{2}+k^{6}+k^{5}+k+1,
\end{aligned}
$$

$$
\begin{aligned}
(\mathrm{A} 8) \quad S_{3}= & b_{1}^{28} k^{9}+b_{1}^{26} k^{7}+b_{1}^{24} k^{9}+b_{1}^{24} k^{8}+b_{1}^{24} k^{7}+b_{1}^{24} k^{6}+b_{1}^{22} k^{6}+b_{1}^{20} k^{11} \\
& +b_{1}^{20} k^{10}+b_{1}^{20} k^{8}+b_{1}^{20} k^{7}+b_{1}^{20} k^{5}+b_{1}^{18} k^{7}+b_{1}^{18} k^{6}+b_{1}^{18} k^{5} \\
& +b_{1}^{18} k^{4}+b_{1}^{16} k^{9}+b_{1}^{16} k^{8}+b_{1}^{16} k^{7}+b_{1}^{16} k^{5}+b_{1}^{16} k^{3}+b_{1}^{14} k^{8} \\
& +b_{1}^{14} k^{7}+b_{1}^{14} k^{6}+b_{1}^{14} k^{4}+b_{1}^{12} k^{8}+b_{1}^{12} k^{3}+b_{1}^{12} k^{2}+b_{1}^{10} k^{6} \\
& +b_{1}^{8} k^{6}+b_{1}^{8} k^{4}+b_{1}^{8}+b_{1}^{4} k^{2}+1, \\
(\mathrm{~A} 9) \quad T_{1}= & a^{8}+a^{6}+a^{5}+a^{2} k+a^{2}+a+1, \\
(\mathrm{~A} 10) \quad T_{2}= & a^{16} k^{3}+a^{16} k^{2}+a^{16} k+a^{16}+a^{14} k^{2}+a^{14}+a^{12}+a^{10} k^{3}+a^{8} k^{4} \\
& +a^{6}+a^{4} k^{2}+a^{4}+a^{2} k^{3}+a^{2} k^{2}+a^{2} k+k^{4}+k^{3}+k^{2}+k+1, \\
\text { (A11) } \quad T_{3}= & a^{26} k^{2}+a^{22} k^{4}+a^{22} k^{2}+a^{20} k^{4}+a^{20} k^{3}+a^{20} k^{2}+a^{18} k^{8}+a^{18} k^{7} \\
& +a^{18} k^{6}+a^{18} k^{4}+a^{16} k^{6}+a^{16} k^{3}+a^{16} k^{2}+a^{14} k^{7}+a^{14} k^{5} \\
& +a^{14} k^{4}+a^{14} k^{2}+a^{12} k^{8}+a^{12} k^{5}+a^{12} k^{3}+a^{12} k^{2}+a^{12} k
\end{aligned}
$$




$$
\begin{aligned}
& +a^{10} k^{9}+a^{10} k^{8}+a^{10} k^{7}+a^{10} k^{5}+a^{10} k^{2}+a^{10} k+a^{10}+a^{8} k^{8} \\
& +a^{8} k^{7}+a^{8} k^{4}+a^{6} k^{5}+a^{6} k^{3}+a^{6} k^{2}+a^{4} k^{8}+a^{4} k^{7}+a^{4} k^{6} \\
& +a^{4} k^{4}+a^{2} k^{9}+a^{2} k^{5}+a^{2} k^{4}+k^{8} .
\end{aligned}
$$

In (3.41) - (3.43),

(A12)

$$
\begin{aligned}
& h_{1}^{*}=a^{33}+a^{31}+a^{29}+a^{27}+\left(a^{61}+a^{60}+a^{59}+a^{58}+a^{57}+a^{56}+a^{53}+a^{52}+a^{51}\right. \\
& +a^{50}+a^{49}+a^{48}+a^{37}+a^{36}+a^{35}+a^{34}+a^{33}+a^{32}+a^{29}+a^{28}+a^{27}+a^{26}+a^{25} \\
& \left.+a^{24}+a^{21}+a^{20}+a^{19}+a^{18}+a^{17}+a^{16}+a^{5}+a^{4}+a^{3}+a^{2}+a+1\right) b_{1}^{19}+\left(a^{66}\right. \\
& +a^{65}+a^{64}+a^{62}+a^{60}+a^{59}+a^{58}+a^{56}+a^{55}+a^{49}+a^{48}+a^{47}+a^{45}+a^{36}+a^{33} \\
& +a^{32}+a^{28}+a^{27}+a^{24}+a^{23}+a^{22}+a^{18}+a^{17}+a^{16}+a^{15}+a^{14}+a^{13}+a^{10}+a^{6} \\
& \left.+a^{4}\right) b_{1}^{18}+\left(a^{67}+a^{66}+a^{60}+a^{59}+a^{58}+a^{57}+a^{55}+a^{54}+a^{52}+a^{51}+a^{50}+a^{49}\right. \\
& +a^{46}+a^{45}+a^{44}+a^{40}+a^{38}+a^{33}+a^{32}+a^{29}+a^{26}+a^{25}+a^{24}+a^{20}+a^{19}+a^{14} \\
& \left.+a^{11}+a^{7}+a^{3}+1\right) b_{1}^{17}+\left(a^{65}+a^{63}+a^{61}+a^{60}+a^{58}+a^{57}+a^{55}+a^{54}+a^{53}+a^{52}\right. \\
& +a^{50}+a^{48}+a^{46}+a^{41}+a^{38}+a^{36}+a^{32}+a^{30}+a^{28}+a^{27}+a^{26}+a^{25}+a^{23}+a^{22} \\
& \left.+a^{15}+a^{13}+a^{11}+a^{9}+a^{6}+a^{5}+a^{2}+a\right) b_{1}^{16}+\left(a^{58}+a^{55}+a^{54}+a^{52}+a^{51}+a^{50}\right. \\
& +a^{49}+a^{47}+a^{46}+a^{44}+a^{42}+a^{41}+a^{38}+a^{36}+a^{33}+a^{32}+a^{30}+a^{28}+a^{27}+a^{26} \\
& \left.+a^{20}+a^{19}+a^{16}+a^{15}+a^{11}+a^{10}+a^{9}+a^{7}+a^{4}+a^{2}\right) b_{1}^{15}+\left(a^{56}+a^{55}+a^{53}+a^{52}\right. \\
& +a^{48}+a^{46}+a^{45}+a^{44}+a^{42}+a^{35}+a^{33}+a^{31}+a^{30}+a^{27}+a^{26}+a^{25}+a^{24}+a^{23} \\
& \left.+a^{22}+a^{21}+a^{20}+a^{18}+a^{17}+a^{15}+a^{14}+a^{13}+a^{11}+a^{10}+a^{9}+a^{8}+a^{4}+a^{3}\right) b_{1}^{14} \\
& +\left(a^{54}+a^{52}+a^{51}+a^{50}+a^{49}+a^{48}+a^{46}+a^{45}+a^{40}+a^{38}+a^{36}+a^{35}+a^{27}+a^{26}\right. \\
& \left.+a^{22}+a^{21}+a^{19}+a^{18}+a^{17}+a^{16}+a^{12}+a^{10}+a^{8}+a^{4}\right) b_{1}^{13}+\left(a^{52}+a^{51}+a^{50}\right. \\
& +a^{48}+a^{47}+a^{41}+a^{37}+a^{36}+a^{35}+a^{34}+a^{33}+a^{30}+a^{29}+a^{28}+a^{27}+a^{24}+a^{13} \\
& \left.+a^{12}+a^{11}+a^{7}+a^{6}+a^{5}\right) b_{1}^{12}+\left(a^{50}+a^{47}+a^{44}+a^{42}+a^{40}+a^{38}+a^{37}+a^{35}\right. \\
& \left.+a^{34}+a^{33}+a^{27}+a^{25}+a^{23}+a^{22}+a^{21}+a^{17}+a^{16}+a^{14}+a^{12}+a^{10}+a^{9}+a^{6}\right) b_{1}^{11} \\
& +\left(a^{48}+a^{47}+a^{45}+a^{43}+a^{42}+a^{33}+a^{32}+a^{31}+a^{29}+a^{27}+a^{26}+a^{20}+a^{17}+a^{16}\right. \\
& \left.+a^{15}+a^{12}+a^{8}+a^{7}\right) b_{1}^{10}+\left(a^{46}+a^{44}+a^{42}+a^{41}+a^{39}+a^{38}+a^{36}+a^{35}+a^{34}\right. \\
& +a^{32}+a^{31}+a^{30}+a^{28}+a^{27}+a^{26}+a^{24}+a^{23}+a^{21}+a^{20}+a^{19}+a^{18}+a^{16}+a^{15} \\
& \left.+a^{14}+a^{12}+a^{11}\right) b_{1}^{9}+\left(a^{44}+a^{43}+a^{42}+a^{41}+a^{39}+a^{38}+a^{37}+a^{36}+a^{34}+a^{32}\right. \\
& \left.+a^{31}+a^{29}+a^{28}+a^{27}+a^{26}+a^{24}+a^{23}+a^{22}+a^{20}+a^{17}+a^{16}+a^{15}+a^{13}+a^{11}\right) b_{1}^{8} \\
& +\left(a^{42}+a^{39}+a^{38}+a^{36}+a^{35}+a^{34}+a^{33}+a^{31}+a^{30}+a^{26}+a^{25}+a^{24}+a^{17}\right) b_{1}^{7} \\
& +\left(a^{40}+a^{39}+a^{37}+a^{36}+a^{32}+a^{30}+a^{29}+a^{28}+a^{26}+a^{20}+a^{19}+a^{18}+a^{17}\right) b_{1}^{6} \\
& +\left(a^{38}+a^{36}+a^{35}+a^{34}+a^{33}+a^{32}+a^{30}+a^{29}+a^{25}+a^{23}+a^{22}+a^{21}+a^{20}\right) b_{1}^{5} \\
& +\left(a^{36}+a^{35}+a^{34}+a^{32}+a^{31}+a^{25}+a^{23}+a^{22}\right) b_{1}^{4}+\left(a^{34}+a^{31}+a^{29}+a^{27}+a^{25}\right. \\
& \left.+a^{22}\right) b_{1}^{3}+\left(a^{34}+a^{33}+a^{31}+a^{30}+a^{29}+a^{28}+a^{24}+a^{23}\right) b_{1}^{2}+\left(a^{35}+a^{34}+a^{30}+a^{27}\right) b_{1},
\end{aligned}
$$


(A13)

$$
\begin{aligned}
& h_{2}^{*}=a^{35}+a^{33}+a^{31}+a^{29}+\left(a^{73}+a^{72}+a^{69}+a^{68}+a^{67}+a^{66}+a^{65}+a^{64}+a^{57}\right. \\
& +a^{56}+a^{53}+a^{52}+a^{51}+a^{50}+a^{45}+a^{44}+a^{43}+a^{42}+a^{41}+a^{40}+a^{25}+a^{24}+a^{21} \\
& \left.+a^{20}+a^{19}+a^{18}+a^{13}+a^{12}+a^{11}+a^{10}+a^{5}+a^{4}+a^{3}+a^{2}+a+1\right) b_{1}^{19}+\left(a^{71}\right. \\
& +a^{69}+a^{65}+a^{55}+a^{53}+a^{49}+a^{47}+a^{45}+a^{41}+a^{23}+a^{21}+a^{17}+a^{15}+a^{13}+a^{9} \\
& \left.+a^{7}+a^{5}+a\right) b_{1}^{18}+\left(a^{69}+a^{68}+a^{64}+a^{63}+a^{60}+a^{59}+a^{56}+a^{55}+a^{53}+a^{51}+a^{45}\right. \\
& +a^{44}+a^{40}+a^{39}+a^{36}+a^{35}+a^{32}+a^{31}+a^{28}+a^{27}+a^{24}+a^{23}+a^{21}+a^{19}+a^{13} \\
& \left.+a^{12}+a^{8}+a^{7}+a^{5}+a^{3}\right) b_{1}^{17}+\left(a^{67}+a^{65}+a^{63}+a^{60}+a^{59}+a^{57}+a^{56}+a^{54}+a^{52}\right. \\
& +a^{51}+a^{50}+a^{49}+a^{47}+a^{44}+a^{42}+a^{41}+a^{40}+a^{39}+a^{37}+a^{32}+a^{29}+a^{23}+a^{22} \\
& \left.+a^{21}+a^{18}+a^{16}+a^{13}+a^{11}+a^{10}+a^{4}\right) b_{1}^{16}+\left(a^{59}+a^{57}+a^{55}+a^{54}+a^{53}+a^{51}\right. \\
& +a^{50}+a^{48}+a^{47}+a^{45}+a^{40}+a^{37}+a^{36}+a^{35}+a^{34}+a^{30}+a^{29}+a^{27}+a^{24}+a^{22} \\
& \left.+a^{21}+a^{20}+a^{16}+a^{15}+a^{14}+a^{9}+a^{7}+a^{5}\right) b_{1}^{15}+\left(a^{57}+a^{56}+a^{55}+a^{54}+a^{49}+a^{47}\right. \\
& +a^{43}+a^{41}+a^{39}+a^{37}+a^{33}+a^{32}+a^{30}+a^{25}+a^{23}+a^{21}+a^{16}+a^{15}+a^{14}+a^{11} \\
& \left.+a^{9}+a^{8}+a^{7}+a^{6}\right) b_{1}^{14}+\left(a^{55}+a^{47}+a^{46}+a^{45}+a^{44}+a^{41}+a^{39}+a^{36}+a^{35}+a^{33}\right. \\
& \left.+a^{32}+a^{31}+a^{29}+a^{26}+a^{25}+a^{23}+a^{20}+a^{19}+a^{17}+a^{16}+a^{14}+a^{12}+a^{10}+a^{7}\right) b_{1}^{13} \\
& +\left(a^{53}+a^{52}+a^{49}+a^{47}+a^{46}+a^{42}+a^{41}+a^{40}+a^{38}+a^{36}+a^{34}+a^{30}+a^{28}+a^{27}\right. \\
& \left.+a^{24}+a^{21}+a^{20}+a^{17}+a^{16}+a^{15}+a^{14}+a^{11}+a^{9}+a^{8}\right) b_{1}^{12}+\left(a^{51}+a^{48}+a^{46}\right. \\
& +a^{45}+a^{44}+a^{41}+a^{37}+a^{35}+a^{34}+a^{32}+a^{30}+a^{27}+a^{25}+a^{19}+a^{16}+a^{12}+a^{10} \\
& \left.+a^{8}\right) b_{1}^{11}+\left(a^{49}+a^{48}+a^{46}+a^{44}+a^{43}+a^{42}+a^{41}+a^{39}+a^{37}+a^{33}+a^{27}+a^{26}\right. \\
& \left.+a^{22}+a^{21}+a^{19}+a^{18}+a^{16}+a^{15}+a^{12}+a^{11}+a^{10}+a^{9}\right) b_{1}^{10}+\left(a^{47}+a^{45}+a^{44}\right. \\
& +a^{43}+a^{40}+a^{39}+a^{37}+a^{35}+a^{31}+a^{30}+a^{29}+a^{27}+a^{20}+a^{19}+a^{16}+a^{15}+a^{14} \\
& \left.+a^{13}\right) b_{1}^{9}+\left(a^{45}+a^{44}+a^{43}+a^{41}+a^{40}+a^{38}+a^{36}+a^{35}+a^{34}+a^{33}+a^{31}+a^{30}\right. \\
& \left.+a^{25}+a^{24}+a^{18}+a^{17}+a^{15}+a^{13}\right) b_{1}^{8}+\left(a^{43}+a^{41}+a^{39}+a^{38}+a^{37}+a^{35}+a^{34}\right. \\
& \left.+a^{32}+a^{31}+a^{29}+a^{28}+a^{22}+a^{21}+a^{18}\right) b_{1}^{7}+\left(a^{41}+a^{40}+a^{39}+a^{38}+a^{33}+a^{31}\right. \\
& \left.+a^{27}+a^{25}\right) b_{1}^{6}+\left(a^{39}+a^{31}+a^{30}+a^{29}+a^{28}+a^{25}+a^{22}\right) b_{1}^{5}+\left(a^{37}+a^{36}+a^{33}+a^{31}\right. \\
& \left.+a^{30}+a^{26}+a^{25}+a^{24}\right) b_{1}^{4}+\left(a^{41}+a^{40}+a^{37}+a^{36}+a^{34}+a^{33}+a^{30}+a^{29}+a^{28}\right. \\
& \left.+a^{24}\right) b_{1}^{3}+\left(a^{39}+a^{37}+a^{32}+a^{30}+a^{28}+a^{27}+a^{26}+a^{25}\right) b_{1}^{2}+\left(a^{37}+a^{36}+a^{32}+a^{29}\right) b_{1},
\end{aligned}
$$

(A14)

$$
\begin{aligned}
& h_{3}^{*}=a^{36}+a^{28}+\left(a^{68}+a^{67}+a^{66}+a^{64}+a^{62}+a^{60}+a^{58}+a^{56}+a^{54}+a^{51}+a^{44}\right. \\
& +a^{43}+a^{42}+a^{40}+a^{38}+a^{36}+a^{34}+a^{32}+a^{30}+a^{28}+a^{26}+a^{24}+a^{22}+a^{19}+a^{12} \\
& \left.+a^{11}+a^{10}+a^{8}+a^{6}+a^{3}\right) b_{1}^{23}+\left(a^{67}+a^{65}+a^{61}+a^{59}+a^{58}+a^{57}+a^{53}+a^{50}+a^{43}\right. \\
& +a^{41}+a^{37}+a^{35}+a^{34}+a^{33}+a^{29}+a^{27}+a^{26}+a^{25}+a^{21}+a^{18}+a^{11}+a^{9}+a^{5} \\
& \left.+a^{2}\right) b_{1}^{22}+\left(a^{68}+a^{67}+a^{66}+a^{65}+a^{64}+a^{61}+a^{60}+a^{59}+a^{57}+a^{56}+a^{55}+a^{54}\right. \\
& +a^{53}+a^{52}+a^{51}+a^{50}+a^{48}+a^{47}+a^{45}+a^{44}+a^{43}+a^{38}+a^{37}+a^{36}+a^{34}+a^{32} \\
& +a^{30}+a^{29}+a^{28}+a^{27}+a^{26}+a^{25}+a^{24}+a^{23}+a^{21}+a^{20}+a^{19}+a^{16}+a^{15}+a^{14}
\end{aligned}
$$




$$
\begin{aligned}
& \left.+a^{13}+a^{12}+a^{11}+a^{10}+a^{5}+a^{3}+a^{2}+a\right) b_{1}^{21}+\left(a^{69}+a^{68}+a^{63}+a^{60}+a^{57}+a^{56}\right. \\
& +a^{55}+a^{50}+a^{49}+a^{47}+a^{43}+a^{41}+a^{40}+a^{38}+a^{37}+a^{36}+a^{35}+a^{32}+a^{30}+a^{28} \\
& \left.+a^{27}+a^{24}+a^{23}+a^{21}+a^{20}+a^{17}+a^{16}+a^{14}+a^{8}+a^{6}+a^{5}+a^{4}+a^{3}+a^{2}\right) b_{1}^{20} \\
& +\left(a^{68}+a^{66}+a^{65}+a^{64}+a^{63}+a^{62}+a^{59}+a^{58}+a^{57}+a^{55}+a^{54}+a^{50}+a^{48}+a^{47}\right. \\
& +a^{45}+a^{42}+a^{40}+a^{37}+a^{36}+a^{33}+a^{31}+a^{29}+a^{28}+a^{25}+a^{24}+a^{21}+a^{20}+a^{15} \\
& \left.+a^{14}+a^{12}+a^{11}+a^{7}+a^{6}+a^{4}\right) b_{1}^{19}+\left(a^{69}+a^{68}+a^{67}+a^{66}+a^{65}+a^{63}+a^{54}+a^{53}\right. \\
& +a^{52}+a^{51}+a^{50}+a^{49}+a^{48}+a^{46}+a^{42}+a^{41}+a^{39}+a^{38}+a^{30}+a^{29}+a^{26}+a^{21} \\
& \left.+a^{20}+a^{19}+a^{15}+a^{13}+a^{9}+a^{7}+a^{5}+a^{4}+a^{3}+1\right) b_{1}^{18}+\left(a^{70}+a^{69}+a^{68}+a^{67}\right. \\
& +a^{66}+a^{64}+a^{62}+a^{61}+a^{60}+a^{59}+a^{58}+a^{55}+a^{54}+a^{52}+a^{48}+a^{47}+a^{45}+a^{44} \\
& +a^{42}+a^{41}+a^{40}+a^{38}+a^{37}+a^{36}+a^{35}+a^{33}+a^{32}+a^{30}+a^{28}+a^{27}+a^{26}+a^{24} \\
& \left.+a^{23}+a^{22}+a^{20}+a^{19}+a^{18}+a^{17}+a^{15}+a^{14}+a^{9}+a^{8}+a^{6}+a^{4}+a^{3}+a^{2}\right) b_{1}^{17} \\
& +\left(a^{68}+a^{64}+a^{63}+a^{62}+a^{60}+a^{58}+a^{53}+a^{52}+a^{50}+a^{46}+a^{42}+a^{40}+a^{39}+a^{36}\right. \\
& +a^{35}+a^{29}+a^{26}+a^{25}+a^{24}+a^{23}+a^{21}+a^{20}+a^{19}+a^{18}+a^{17}+a^{15}+a^{14}+a^{13} \\
& \left.+a^{12}+a^{11}+a^{10}+a^{8}+a^{6}+a^{3}\right) b_{1}^{16}+\left(a^{62}+a^{59}+a^{58}+a^{54}+a^{52}+a^{48}+a^{47}+a^{42}\right. \\
& +a^{40}+a^{39}+a^{38}+a^{33}+a^{31}+a^{30}+a^{29}+a^{26}+a^{20}+a^{18}+a^{17}+a^{14}+a^{13}+a^{12} \\
& \left.+a^{11}+a^{7}+a^{6}+a^{4}\right) b_{1}^{15}+\left(a^{60}+a^{59}+a^{55}+a^{54}+a^{53}+a^{48}+a^{44}+a^{42}+a^{41}+a^{40}\right. \\
& +a^{39}+a^{38}+a^{37}+a^{34}+a^{32}+a^{30}+a^{29}+a^{28}+a^{27}+a^{26}+a^{23}+a^{21}+a^{18}+a^{16} \\
& \left.+a^{13}+a^{12}+a^{9}+a^{7}+a^{6}+a^{5}\right) b_{1}^{14}+\left(a^{58}+a^{56}+a^{52}+a^{50}+a^{49}+a^{47}+a^{46}+a^{44}\right. \\
& +a^{43}+a^{39}+a^{38}+a^{33}+a^{32}+a^{28}+a^{26}+a^{24}+a^{22}+a^{21}+a^{19}+a^{18}+a^{16}+a^{13} \\
& \left.+a^{12}+a^{6}\right) b_{1}^{13}+\left(a^{56}+a^{55}+a^{54}+a^{49}+a^{47}+a^{44}+a^{42}+a^{41}+a^{39}+a^{38}+a^{37}\right. \\
& +a^{36}+a^{34}+a^{33}+a^{30}+a^{29}+a^{28}+a^{27}+a^{26}+a^{22}+a^{18}+a^{17}+a^{15}+a^{14}+a^{12} \\
& \left.+a^{10}+a^{8}+a^{7}\right) b_{1}^{12}+\left(a^{54}+a^{51}+a^{49}+a^{48}+a^{46}+a^{44}+a^{42}+a^{40}+a^{30}+a^{26}\right. \\
& \left.+a^{25}+a^{24}+a^{21}+a^{19}+a^{16}+a^{14}+a^{12}+a^{11}+a^{10}+a^{8}\right) b_{1}^{11}+\left(a^{52}+a^{51}+a^{48}\right. \\
& +a^{47}+a^{46}+a^{45}+a^{43}+a^{40}+a^{37}+a^{35}+a^{33}+a^{32}+a^{31}+a^{25}+a^{22}+a^{21}+a^{20} \\
& \left.+a^{19}+a^{18}+a^{16}+a^{14}+a^{12}+a^{10}+a^{9}+a^{8}\right) b_{1}^{10}+\left(a^{50}+a^{48}+a^{42}+a^{41}+a^{40}\right. \\
& +a^{38}+a^{33}+a^{32}+a^{31}+a^{30}+a^{29}+a^{28}+a^{27}+a^{24}+a^{23}+a^{22}+a^{21}+a^{19}+a^{16} \\
& \left.+a^{13}+a^{12}+a^{11}\right) b_{1}^{9}+\left(a^{48}+a^{47}+a^{46}+a^{42}+a^{39}+a^{36}+a^{33}+a^{32}+a^{29}+a^{27}\right. \\
& \left.+a^{26}+a^{23}+a^{20}+a^{19}+a^{15}+a^{14}+a^{12}\right) b_{1}^{8}+\left(a^{46}+a^{43}+a^{42}+a^{38}+a^{37}+a^{36}\right. \\
& \left.+a^{34}+a^{27}+a^{25}+a^{23}+a^{21}+a^{19}+a^{18}+a^{17}\right) b_{1}^{7}+\left(a^{44}+a^{43}+a^{39}+a^{38}+a^{37}\right. \\
& \left.+a^{32}+a^{30}+a^{29}+a^{27}+a^{23}+a^{18}\right) b_{1}^{6}+\left(a^{42}+a^{40}+a^{35}+a^{33}+a^{30}+a^{29}+a^{28}\right. \\
& \left.+a^{23}\right) b_{1}^{5}+\left(a^{40}+a^{39}+a^{38}+a^{37}+a^{36}+a^{33}+a^{31}+a^{29}+a^{25}+a^{22}\right) b_{1}^{4}+\left(a^{38}\right. \\
& \left.+a^{36}+a^{35}+a^{34}+a^{31}+a^{29}+a^{25}+a^{24}\right) b_{1}^{3}+\left(a^{37}+a^{34}+a^{33}+a^{32}+a^{30}+a^{29}\right. \\
& \left.+a^{25}+a^{24}\right) b_{1}^{2}+\left(a^{38}+a^{37}+a^{36}+a^{35}+a^{30}+a^{29}+a^{28}+a^{27}\right) b_{1} \text {. }
\end{aligned}
$$


Department of Mathematics and Statistics, University of South Florida, Tampa, FL 33620

E-mail address: xhou@usf.edu 